\title{
Microscopic dynamics of molecular liquids and glasses: Role of orientations and translation-rotation coupling
}

\author{
T. Theenhaus, R. Schilling \\ Institut für Physik, Johannes Gutenberg-Universität, Staudinger Weg 7, D-55099 Mainz, Germany \\ A. Latz * \\ Institut für Physik, Reichenhainer Strasse 70, TU - Chemnitz, D-09107 Chemnitz, Germany \\ M. Letz \\ Schott Glas, Research and Developement, Hattenbergstr. 10, 55014 Mainz, Germany
}

We investigate the dynamics of a fluid of dipolar hard spheres in its liquid and glassy phase, with emphasis on the microscopic time or frequency regime. This system shows rather different glass transition scenarios related to its rich equilibrium behavior which ranges from a simple hard sphere fluid to a long range ferroelectric orientational order. In the liquid phase close to the ideal glass transition line and in the glassy regime a medium range orientational order occurs leading to a softening of an orientational mode. To investigate the role of this mode we use the molecular mode-coupling equations to calculate the spectra $\phi_{l m}^{\prime \prime}(q, \omega)$ and $\chi_{l m}^{\prime \prime}(q, \omega)$. In the center of mass spectra $\phi_{00}^{\prime \prime}(q, \omega)$ and $\chi_{00}^{\prime \prime}(q, \omega)$ we found besides a high frequency peak at $\omega_{h f}$ a peak at $\omega_{\text {op }}$, about one decade below $\omega_{h f}$. $\omega_{o p}$ has almost no $q$-dependence and exhibits an "isotope" effect $\omega_{o p} \propto I^{-1 / 2}$, with $I$ the moment of inertia. We give evidence that the existence of this peak is related to the occurrence of the medium ranged orientational order. It is shown that some of these feature also exist for schematic mode coupling models.

61.25.Em, 64.70.Pf, 61.20.Lc

\section{INTRODUCTION}

The dynamical properties of liquids and glasses still are a challenging problem. In the vicinity of the glass transition the frequency (or time) range can be decomposed into at least three different domains, the $\alpha, \beta$ and microscopic regime. The first one describes the structural relaxation which dramatically slows down when the glass transition is approached from above. In the idealized mode-coupling theory (MCT) for simple liquids [1 4 , and for molecular systems [5] it even stops at a critical temperature $T_{c}$. Probably the most interesting result of MCT is the existence of the so-called $\beta$-relaxation which describes the dynamics within a cage of particles above and below $T_{c}$. The corresponding $\beta$-frequency scale is much larger than that for the $\alpha$-relaxation. At still higher frequencies there are vibrational and librational motion which constitute the microscopic regime.

One may say that most of the attention in the field of glassy dynamics during the last fifteen years has been devoted to the $\alpha$ - and $\beta$-relaxation. This activity has been mainly stimulated by MCT which has predicted in these two regimes scaling laws with a diverging $\alpha$ - and $\beta$-time scale. These predictions have been tested intensively by experiments and numerical simulations. A satisfactory agreement has been found for many glass forming systems 8 11, 15, 12, 13, , 1, 14. Experimental and simulational results do not exhibit any singular or crossover behavior for microscopic frequencies $\omega$, i.e. for $\omega \geq 1$ THZ. Nevertheless in that regime an interesting phenomenon occurs in most glasses but not in crystals and colloidal glasses, which is the so called boson-peak. Indications for this peak came from two different sides. First, the low temperature specific heat $c(T) / c_{D}(T)$ scaled by the phonon contribution $c_{D}(T) \propto T^{3}$ shows a peak at about $10 \mathrm{~K}$ for several glass formers (see e.g. 116]). This excess with respect to $c_{D}(T)$ around $10 \mathrm{~K}$ implies the existence of additional excitations besides the long wavelength acoustic phonons. Second, Raman spectra $I(\omega)$ compared to the phonon contribution $I_{D}(\omega) \propto \omega^{2}$ exhibit at about 1 THZ an excess, as well (see e.g. 17]). Since the temperature dependence of the excess intensity scales with the Bose-distribution function $n_{B}(T)$, the peak is called boson-peak.

That these two observations might have a common origin was first shown by Buchenau et al. [18,19. For vitreous silica these authors also found an excess with respect to $g_{D}(\omega) \propto \omega^{2}$ for the vibrational density of states $g(\omega)$ determined from inelastic neutron scattering (INS) data. Using $g(\omega)$ to calculate $c(T) / c_{D}(T)$ led to a good agreement with the result from heat capacity measurements. Particularly, the peak positions for both results coincided. Although the boson-peak does not seem to posses any singular $\omega$ - or $T$-dependence it is a universal phenomenon for all systems with exclusion of glass forming colloids, in the sense that it appears more or less for almost all glass formers. For, e.g. LiCl solutions [11] and Orthoterphenyl [20], it has been stressed that the bosonpeak, together with the narrowing of a central peak at $\omega=0$, develops continously from the liquid to the glassy phase.

Despite considerable experimental and numerical efforts its microscopic origin is still not satisfactorily understood. Two reasons might be responsible for that. First, e.g. in case of light scattering the precise connection 
between the measured quantity and the basic theoretical objects, the time- or frequency-dependent site-site or molecular correlation functions for molecular liquids, is not known. For instance, it has been shown that several coupling mechanism between light and distinct modes of liquid $\mathrm{ZnCl}_{2}$ exist which have different $\omega$-dependent coupling constants 21]. This may complicate the determination of $g(\omega)$ from Raman spectra. Second, the relationship between the various $t$ - or $\omega$-dependent correlators and microscopic modes obtained from a diagonalization of the dynamical matrix is not obvious. In addition, these correlators can only be calculated analytically under serious approximations. Apparently, numerical investigations represent a powerful tool since their microscopic nature allows to calculate both, the correlators or spectra and under certain conditions the microscopic modes. The possible character of these modes range between:

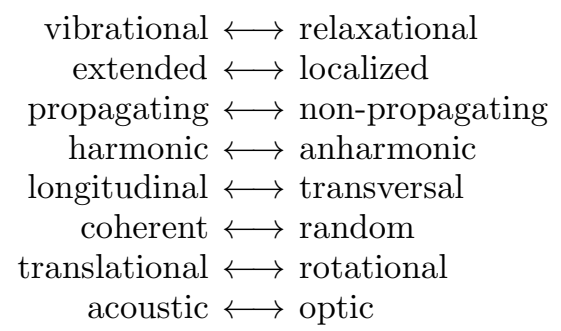

Since these feature can occur in combinations, the complexity of the problem becomes obvious. In addition, different experimental approaches to a specific material or a specific measurement of different type of glass formers, e.g. strong or fragile ones, may exhibit different features of the same phenomenon.

Without demanding completeness let us shortly review the present status. Inelastic X-ray scattering (IXS) on $\mathrm{v}-\mathrm{SiO}_{2}$ gave evidence that propagating acoustic sound waves exist even above $\omega_{B P}$, the position of the bosonpeak [22]. This has let these authors to conclude that the propagating modes are also involved in the boson-peak itself. On the other side a crossover at $\omega_{B P}$ from propagating to localized (strongly scattered) acoustic modes was deduced for $\mathrm{v}_{-} \mathrm{SiO}_{2}$ from INS and IXS [23]. Comments on this controversy are given in Ref. 24 26. However, interpretation of IXS-experiments which are free of any model have recently strengthened at least the fact that there are propagating modes above $\omega_{B P}$ [27,28. MD-simulations for $\mathrm{v}-\mathrm{SiO}_{2}$ have given evidence that the boson-peak modes can not strongly be localized and that there is a contribution of transverse propagating modes in the boson-peak regime 22, 30. IXS for LiCl-solutions and glycerol seem to detect propagating, longitudinal modes at $\omega_{B P}$ [31], whereas a MD-simulation for $\mathrm{H}_{2} \mathrm{O}$ shows a mixing of propagating longitudinal and transversal modes, at least for $q$ large enough $\left(q>4 n m^{-1}\right) 32$.

Further important information about the nature of the boson-peak modes comes from a normal mode analysis for a system of soft spheres [33] and for $\mathrm{SiO}_{2}$ deep in its glass phase [34 37]. There it has been found that these modes are harmonic and quasi localized [33,36. They occur due to hybridization of localized low frequency optic modes with propagating acoustic states [34] and are extended but non-propagating. In addition each normal mode has a coherent and a random component. The latter finding is consistent with other numerical results for a Lennard-Jones liquid [38], liquid $\mathrm{ZnCl}_{2}$ 21, 39,40] and conclusions drawn from experimental data for various glass formers 41,42]. Since $\mathrm{ZnCl}_{2}$ has been considered in its liquid phase an instantaneous normal mode analysis is feasible, only. Restriction to the stable modes accurately reproduces in the microscopic frequency regime the dynamical structure factor $S(q, \omega)$ obtained from a MDsimulation 40 . This implies a harmonic mode character at temperatures even above the MCT glass-transition temperature $T_{c}$.

There is not much analytical work on the boson peak. For a harmonic crystal with random spring constants it has been shown 43, 44, that an excess density of states follows. It has recently been stressed [45] that the approach in 44 has some shortcomings which may be removed by use of off-lattice models [46]. Another theoretical framework is the soft potential model [47 50] which allows to describe the low temperature anomalies below $1 \mathrm{~K}$ and those at 10-20 K, which includes the excess density of states.

As already mentioned above, MCT has been rather successful in describing the $\alpha$ - and $\beta$-dynamics. Schematic MCT-models [1] where the wave number dependence has been neglected were also used to describe experimental spectra in the microscopic regime, including the boson peak 11,51,56. Whereas the results in Ref. [11] (cf. Fig. 6) and Ref. 53] (cf. Fig. 4) yield spectra which are reminiscent to boson peak spectra the line shape does not come out, satisfactorily. However, recently a detailed MCT-investigation for a glass of hard spheres was performed, including the $q$ - dependence [57]. Besides a high frequency peak at $\omega_{H F}$ an additional peak at $\omega_{A O P}$, about one decade below $\omega_{H F}$, has been seen for a volume fraction $\varphi=0.6$. This peak, which strongly resembles that in Refs. [11] (Fig. 6) and [52] (Fig. 7), originates from the distribution of harmonic oscillators within the cages and has been called anomalous oscillation peak (AOP). As explicitely demonstrated [57] it shares many features with the boson peak.

In contrast to a system of hard spheres or binary van der Waals liquids, molecular liquids also have orientational degrees of freedom. The question which one may ask is: Does the boson peak also involve orientational motion? Indeed one of its first interpretations for $\mathrm{v}-\mathrm{SiO}_{2}$ were coupled rotations of $\mathrm{SiO}_{4}$-tetrahedra [18]. Such an interpretation has been supported by MD-simulations for $\mathrm{v}_{-} \mathrm{SiO}_{2}$ [35,58] and $\mathrm{ZnCl}_{2}$ [40] and by neutron scattering experiments, proving non-sound-like contributions around $\omega_{B P}$ [59]. Dielectric loss measurements probe the orientational dynamics, only. Since these measure- 
ments, e.g. for Glycerol and Propylene Carbonate, also exhibit a boson peak 60,61 gives additional evidence that this peak may also be related to the orientational degrees of freedom. The role of orientational modes becomes even more clear from the experiments on ethanol 62,63. Around $100 \mathrm{~K}$, Ethanol can occur in several phases: glass phase, orientational glass phase, crystalline phase and a rotator phase. The center of mass positions of the molecules are frozen in an amorphous structure for the glass phase, and in a crystalline structure for the three other phases. The orientational dynamic is non-ergodic for the glass and the orientational glass and ergodic for the crystal and the rotator phase. INS has shown the existence of a boson peak for the orientational glass which does not differ much from that in the glass. Even in the rotator phase there is a boson peak, however, shifted to lower frequencies. Similarly to structural glasses the orientational glass phase also exhibits an excess in the specific heat $c(T) / c_{D}(T)$ 62. These findings suggest that these excess modes are primarily related to the orientational degrees of freedom.

In the present paper we will not calculate the density of states, but the susceptibility and correlation spectra of the collective dynamics. These quantities show the appearance of an extra peak about a decade below the high frequency peak, where the former also originates from the orientational degrees of motion.

The outline of the paper is as follows. In Sec. III, we will shortly review the derivation of the equation of motion for the relevant correlators of rigid, linear molecules and the mode-coupling approximation. In addition, we will discuss the linearized equations. The molecular mode-coupling equations are solved numerically for a liquid of dipolar hard spheres in Sec. IID. It is shown in Sec. III that the qualitative features of the susceptibility spectra obtained for dipolar hard spheres can be derived from a schematic model. Finally, Section IV] contains a discussion of the results and some conclusions.

\section{EQUATIONS OF MOTION}

In the first part of this section we will present the equations of motion for the most relevant correlation functions of a molecular liquid. The second part contains a discussion of the corresponding linearized equations, which yields information on the microscopic time scale and in the third part we will shortly review the mode-coupling approximation.

\section{A. Mori equation}

We restrict ourselves to a system of $N$ rigid and linear molecules with mass $M$ and moment of inertia $I$. There are two possibilities to describe molecular liquids: a site-site or a molecular representation 64. The latter, which will be chosen here, decomposes the $5 N$ degrees of freedom into $3 N$ translational and $2 N$ orientational ones. The starting point is the microscopic tensorial oneparticle density mode

$$
\rho_{l m}(\vec{q}, t)=\sqrt{4 \pi} i^{l} \sum_{n=1}^{N} e^{i \vec{q} \cdot \vec{x}_{n}(t)} Y_{l m}\left(\Omega_{n}(t)\right)
$$

and the corresponding translational and rotational current density mode

$$
\vec{j}_{l m}^{\alpha}(\vec{q}, t)=\sqrt{4 \pi} i^{l} \sum_{n=1}^{N} \vec{v}_{n}^{\alpha}(t) e^{i \vec{q} \cdot \vec{x}_{n}(t)} Y_{l m}\left(\Omega_{n}(t)\right)
$$

for $\alpha=T$ and $R$, respectively. $\vec{x}_{n}(t)$ and $\Omega_{n}(t)=$ $\left(\theta_{n}(t), \varphi_{n}(t)\right)$ are the center of mass position and the orientation (specified by the two angles $\theta_{n}$ and $\varphi_{n}$ ) of the $n$-th molecule at time $t$, respectively. $Y_{l m}(\theta, \varphi)$ are the spherical harmonics with $l=0,1,2, \ldots,-l \leq m \leq l$ and the velocities $\vec{v}_{n}^{\alpha}(t)$ are defined by

$$
\vec{v}_{n}^{\alpha}(t)= \begin{cases}\overrightarrow{\dot{x}}_{n}(t) & , \quad \alpha=T \\ \vec{\omega}_{n}(t) & , \quad \alpha=R\end{cases}
$$

where $\vec{\omega}_{n}(t)$ is the corresponding angular velocity. $\rho_{l m}$ and $\vec{j}_{l m}^{\alpha}$ are related to each other by the continuity equation:

$$
\dot{\rho}_{l m}(\vec{q}, t)=i \sum_{\alpha=T, R} q_{l}^{\alpha}(\vec{q}) j_{l m}^{\alpha}(\vec{q}, t)
$$

with:

$$
\begin{aligned}
& j_{l m}^{T}(\vec{q}, t)=\frac{1}{q} \vec{q} \cdot \vec{j}_{l m}^{T}(\vec{q}, t), \quad q=|\vec{q}| \\
& j_{l m}^{R}(\vec{q}, t)=\frac{1}{\sqrt{l(l+1)}} \vec{L} \cdot \vec{j}_{l m}^{R}(\vec{q}, t)
\end{aligned}
$$

the "longitudinal" translational and rotational current density and

$$
q_{l}^{\alpha}(\vec{q}):=\left\{\begin{array}{ccc}
q & , \quad \alpha=T, & \forall l \\
\sqrt{l(l+1)} & , \quad \alpha=R, & \forall \vec{q} .
\end{array}\right.
$$

$\vec{L}$ denotes the operator of angular momentum.

The time- and $\left(\vec{q} ; l m, l^{\prime} m^{\prime}\right)$-dependent correlation functions:

$$
S_{l m, l^{\prime} m^{\prime}}(\vec{q}, t)=\frac{1}{N}\left\langle\delta \rho_{l m}^{*}(\vec{q}, t) \delta \rho_{l^{\prime} m^{\prime}}(\vec{q}, 0)\right\rangle
$$

and

$$
J_{l m, l^{\prime} m^{\prime}}^{\alpha \alpha^{\prime}}(\vec{q}, t)=\frac{1}{N}\left\langle j_{l m}^{\alpha *}(\vec{q}, t) j_{l^{\prime} m^{\prime}}^{\alpha^{\prime}}(\vec{q}, 0)\right\rangle
$$

are of particular experimental and theoretical importance, at least for $l=l^{\prime} \leq 2$. Here, $\delta \rho_{l m}(\vec{q}, t)=$ $\rho_{l m}(\vec{q}, t)-\left\langle\rho_{l m}(\vec{q}, t)\right\rangle$ is the corresponding fluctuation of the density. $\mathbf{S}(\vec{q}, t)=\left(S_{l m, l^{\prime} m^{\prime}}(\vec{q}, t)\right)$ form a complete 
set for any time dependent two-point density correlator. Particularly, the partial dynamical structure factors in a site-site description are linear superpositions of the molecular correlators $S_{l m, l^{\prime} m^{\prime}}(\vec{q}, t)$, but not vice versa [65]. In addition, we introduce the density-current density correlator:

$$
S_{l m, l^{\prime} m^{\prime}}^{\alpha}(\vec{q}, t)=\frac{1}{N}\left\langle j_{l m}^{\alpha *}(\vec{q}, t) \delta \rho_{l^{\prime} m^{\prime}}(\vec{q}, 0)\right\rangle
$$

Mori-Zwanzig formalism has been used to derive equations of motion for $\mathbf{S}(\vec{q}, t)$ for a single linear molecule in a liquid of isotropic particles [5] and for a molecular liquid of linear [6] and arbitrary molecules [7]. Similar work has been done in a site-site description [66. A comparison between the tensorial and the site-site mode coupling theory has recently been performed for a single dumbell in a liquid of hard spheres 67].

Choosing $\delta \rho_{l m}$ and both scalar current densities $j_{l m}^{\alpha}, \alpha=T, R$ as slow variables, the Mori-Zwanzig formalism leads to the following set of equations:

$$
\begin{array}{r}
\dot{\mathbf{S}}(\vec{q}, t)+i \sum_{\alpha} \mathbf{q}^{\alpha} \mathbf{S}^{\alpha}(\vec{q}, t)=0 \\
\dot{\mathbf{S}}^{\alpha}(\vec{q}, t)+i \mathbf{q}^{\alpha} \mathbf{J}^{\alpha}(\vec{q}) \mathbf{S}^{-1}(\vec{q}, 0) \mathbf{S}(\vec{q}, t)+ \\
\mathbf{J}^{\alpha}(\vec{q}) \int_{0}^{t} d t^{\prime} \sum_{\alpha^{\prime}} \mathbf{m}^{\alpha \alpha^{\prime}}\left(\vec{q}, t-t^{\prime}\right) \mathbf{S}^{\alpha^{\prime}}\left(\vec{q}, t^{\prime}\right)=0
\end{array}
$$

with initial conditions:

$$
\begin{aligned}
\mathbf{S}(\vec{q}, 0) & \equiv \mathbf{S}(\vec{q}) \\
\dot{\mathbf{S}}(\vec{q}, 0) & \equiv 0 \equiv \mathbf{S}^{\alpha}(\vec{q}, 0) \\
\dot{\mathbf{S}}^{\alpha}(\vec{q}, 0) & \equiv-i \mathbf{J}(\overrightarrow{\mathbf{q}})^{\alpha} \mathbf{q}^{\alpha}
\end{aligned}
$$

and

$$
\begin{aligned}
\left(\mathbf{q}^{\alpha}\right)_{l m, l^{\prime} m^{\prime}} & =q_{l}^{\alpha}(\vec{q}) \delta_{l l^{\prime}} \delta_{m m^{\prime}} \\
\left(\mathbf{J}^{\alpha}(\vec{q})\right)_{l m, l^{\prime} m^{\prime}} & \equiv J_{l m, l^{\prime} m^{\prime}}^{\alpha \alpha^{\prime}}(\vec{q})=\frac{k_{B} T}{I^{\alpha}} \delta_{\alpha \alpha^{\prime}} \delta_{l l^{\prime}} \delta_{m m^{\prime}}
\end{aligned}
$$

Here, $\mathbf{S}(\vec{q})$ and $\mathbf{J}^{\alpha \alpha^{\prime}}(\vec{q})$ denote, respectively, the static density and current density correlation matrix and

$$
I_{\alpha}=\left\{\begin{array}{cc}
M, & \alpha=T \\
I, & \alpha=R
\end{array}\right.
$$

Note first that these equations are completely equivalent to the set of equations in [6]. However, they are given in a different representation, which makes its numerical solution convenient. Second, this set of equations is still exact, but needs an expression for the memory kernels $m_{l m, l^{\prime} m^{\prime}}^{\alpha \alpha^{\prime}}(\vec{q}, t)$. This is where approximations come in. Their nature depends strongly on the physical situation: for example $\mathbf{m}^{\alpha \alpha^{\prime}}$ for a supercooled liquid will be quite different from that for a liquid at higher temperatures. Third, instead of choosing the scalar current densities one could also use each Cartesian component $j_{l m}^{\alpha i}, i=x, y, z$ as slow variables. This has been done recently for $\alpha=T$, but not for $\alpha=R$ in order to discuss the role of transversal currents on light scattering spectra [68. The resulting equations again are exact but involve memory kernels $m_{l m, l^{\prime} m^{\prime}}^{\alpha i, \alpha^{\prime} i}(\vec{q}, t)$.

\section{B. Linearized equations of motion}

Since the memory kernels will not be independent functions of the tensorial density correlations $\mathbf{S}(\overrightarrow{\mathbf{q}}, \mathbf{t})$, the third term in Eq. (10b) is a kind of nonlinearity. Within $\operatorname{MCT} \mathbf{m}^{\alpha \alpha^{\prime}}(\overrightarrow{\mathbf{q}}, \mathbf{t})$ is approximated by superpositions of products $\mathbf{S}\left(\overrightarrow{\mathbf{q}}_{\mathbf{1}}, \mathbf{t}\right) \mathbf{S}\left(\overrightarrow{\mathbf{q}}_{\mathbf{2}}, \mathbf{t}\right)$ with $\vec{q}=\vec{q}_{1}+\vec{q}_{2}$, which makes obvious the nonlinear character of the equations of motion. It is this nonlinearity which leads to a slowing down of the structural relaxation by decreasing the temperature or increasing the density of a liquid. This behavior takes place on the liquid side as a two-step relaxation process characterized by two diverging time scales $t_{\sigma} \sim\left|T-T_{c}\right|^{-\frac{1}{2 a}}$ and $\tau \sim\left(T-T_{c}\right)^{-\gamma}$ where $a$ and $\gamma$ are positive and $T_{c}$ is the ideal glass transition temperature. The time scale for $t_{\sigma}$ and $\tau$ is determined by a microscope scale $t_{0} . t_{0}$ depends on inertia and damping effects where the latter are due to the regular part of $\mathbf{m}^{\alpha \alpha^{\prime}}(\overrightarrow{\mathbf{q}}, \mathbf{t})$, accounting for the fast motions. Because our main interest is the microscopic dynamics, we can get an estimate of the microscopic time scale by neglecting the memory term including its regular part. This results in a set of linear equations from which one immediately obtains for the normalized correlator $\mathbf{\Phi}(\vec{q}, t)=\mathbf{S}^{-\frac{1}{2}}(\overrightarrow{\mathbf{q}}) \mathbf{S}(\overrightarrow{\mathbf{q}}, \mathbf{t}) \mathbf{S}^{-\frac{1}{2}}(\overrightarrow{\mathbf{q}})$ :

$$
\ddot{\boldsymbol{\Phi}}(\vec{q}, t)+\mathbf{\Omega}^{2}(\vec{q}) \boldsymbol{\Phi}(\vec{q}, t)=0
$$

with initial conditions

$$
\boldsymbol{\Phi}(\vec{q}, 0)=\mathbf{1}, \quad \dot{\boldsymbol{\Phi}}(\vec{q}, 0)=\mathbf{0} .
$$

and the hermitian frequency matrix squared:

$$
\boldsymbol{\Omega}^{2}(\vec{q})=\mathbf{S}^{-\frac{1}{2}}(\vec{q}) \sum_{\alpha}\left(\mathbf{q}^{\alpha}\right)^{2} \mathbf{J}^{\alpha} \mathbf{S}^{-\frac{1}{2}}(\vec{q})
$$

or with Eq. (6), (14) and (16)

$$
\begin{aligned}
\left(\mathbf{\Omega}^{2}(\vec{q})\right)_{l m \cdot l^{\prime} m^{\prime}}= & \sum_{l^{\prime \prime} m^{\prime \prime}}\left(\mathbf{S}^{-\frac{1}{2}}(\vec{q})\right)_{l m, l^{\prime \prime} m^{\prime \prime}} \\
& \times\left[\frac{k_{B} T}{M} q^{2}+\frac{k_{B} T}{I} l^{\prime \prime}\left(l^{\prime \prime}+1\right)\right] \\
& \times\left(\mathbf{S}^{-\frac{1}{2}}(\vec{q})\right)_{l^{\prime \prime} m^{\prime \prime}, l^{\prime} m^{\prime}}
\end{aligned}
$$

Here, some comments are in order. First, the static correlators $S_{l m, l^{\prime} m^{\prime}}(\vec{q})$ and therefore $\Omega_{l m, l^{\prime} m^{\prime}}(\vec{q})$ are not diagonal in $l$ and $l^{\prime}$ in general. Accordingly, translational and rotational modes generally are coupled to each other for given $\vec{q}$. Second, this coupling vanishes in the limit $q \rightarrow 0$, because the static correlators $S_{l m, l^{\prime} m^{\prime}}(\vec{q})$ and 
therefore $\Omega_{l m, l^{\prime} m^{\prime}}(\vec{q})$ (cf. Eq. (20)) become diagonal and independent from each other for an isotropic liquid:

$$
\Omega_{l m, l^{\prime} m^{\prime}}(\vec{q}) \rightarrow \omega_{l}(q) \delta_{l l^{\prime}} \delta_{m m^{\prime}}
$$

with

$$
\begin{aligned}
\omega_{0}(q) & =\sqrt{\frac{k_{B} T}{M S_{0}}} q \\
\omega_{l}(q) & =\sqrt{\frac{k_{B} T}{I S_{l}}} \sqrt{l(l+1)}, \quad l>0
\end{aligned}
$$

the translational and rotational frequencies for $q \rightarrow 0$ and $S_{l} \equiv S_{l 0, l 0}(\vec{q}=0)$. $\omega_{0}(q)$ describes the well known acoustic isothermal sound wave dispersion and $\omega_{l}(q)$ the optic rotational frequencies for $l>0$.

Third, the limit $q \rightarrow 0$ was already discussed for a molecular liquid using a site-site description 69. These authors also have set the corresponding memory matrix to zero, which, by the way, is completely equivalent to a short time expansion of the equation of motion in leading order. But there the coupling between the partial dynamical structure factors does not vanish for $q \rightarrow 0$. In this respect the choice of a molecular representation which takes care of the isotropy of the microscopic Hamiltonian is the most natural one, at least for $q \rightarrow 0$ and $t \rightarrow 0$.

So far we have considered the liquid phase only. The idealized version of MCT, which neglects so-called hopping processes, is a theory developed on the liquid side of the glass transition, because it uses as an input static correlators in equilibrium. It may also be used below, but close to the transition point where the singular behavior still dominates. Whether MCT is even capable to describe dynamical feature far below the glass transition point is unclear. Nevertheless, we will also apply MCT for parameters deeper in the glass phase. An ideal glass is a non-ergodic phase with non-ergodicity parameters (not normalized):

$$
F_{\ell m, \ell^{\prime} m^{\prime}}(\vec{q})=\lim _{t \rightarrow \infty} S_{\ell m, \ell^{\prime} m^{\prime}}(\vec{q}, t)
$$

which are nonzero. One can easily prove that Eqs. (10) then imply that

$$
C_{\ell m, \ell^{\prime} m^{\prime}}^{\alpha \alpha^{\prime}}(\vec{q})=\lim _{t \rightarrow \infty} m_{\ell m, \ell^{\prime} m^{\prime}}^{\alpha \alpha^{\prime}}(\vec{q}, t)
$$

are nonzero, too, and that

$$
\sum_{\alpha, \alpha^{\prime}} \mathbf{q}^{\alpha}\left[\left(\mathbf{C}^{\beta \beta^{\prime}}(\vec{q})\right)^{-1}\right]^{\alpha \alpha^{\prime}} \mathbf{q}^{\alpha^{\prime}}=(\mathbf{S}(\vec{q})-\mathbf{F}(\vec{q})) \mathbf{F}^{-1}(\vec{q}) \mathbf{S}(\vec{q}) .
$$

Therefore, we introduce $\hat{\mathbf{S}}(\vec{q}, t)$ and $\hat{\mathbf{m}}^{\alpha \alpha^{\prime}}(\vec{q}, t)$ such that

$$
\begin{aligned}
\mathbf{S}(\vec{q}, t) & =\mathbf{F}(\vec{q})+\hat{\mathbf{S}}(\vec{q}, t) \\
\mathbf{m}^{\alpha \alpha^{\prime}}(\vec{q}, t) & =\mathbf{C}^{\alpha \alpha^{\prime}}(\vec{q})+\hat{\mathbf{m}}^{\alpha \alpha^{\prime}}(\vec{q}, t)
\end{aligned}
$$

in analogy to the approach in 57. Substitution of Eq. (28) into Eq. (10) yields:

$$
\begin{array}{r}
\dot{\hat{\mathbf{S}}}(\vec{q}, t)+i \sum_{\alpha} \mathbf{q}^{\alpha} \mathbf{S}^{\alpha}(\vec{q}, t)=0 \\
\dot{\mathbf{S}}^{\alpha}(\vec{q}, t)+i \mathbf{q}^{\alpha} \mathbf{J}^{\alpha}(\vec{q}) \mathbf{S}^{-1}(\vec{q}) \hat{\mathbf{S}}(\vec{q}, t)+ \\
\mathbf{J}^{\alpha}(\vec{q}) \int_{0}^{t} d t^{\prime} \sum_{\alpha^{\prime}} \hat{\mathbf{m}}^{\alpha \alpha^{\prime}}\left(\vec{q}, t-t^{\prime}\right) \mathbf{S}^{\alpha^{\prime}}\left(\vec{q}, t^{\prime}\right)+ \\
+i \mathbf{q}^{\alpha} \mathbf{J}^{\alpha}(\vec{q}) \mathbf{S}^{-1}(\vec{q}) \mathbf{F}(\vec{q})+ \\
\mathbf{J}^{\alpha}(\vec{q}) \sum_{\alpha^{\prime}} \mathbf{C}^{\alpha \alpha^{\prime}}(\vec{q}) \int_{0}^{t} d t^{\prime} \mathbf{S}^{\alpha^{\prime}}\left(\vec{q}, t^{\prime}\right)=0
\end{array}
$$

with initial conditions:

$$
\hat{\mathbf{S}}(\vec{q}, 0)=\mathbf{S}(\vec{q})-\mathbf{F}(\vec{q})
$$

and

$$
\lim _{t \rightarrow \infty} \hat{\mathbf{S}}(\vec{q}, t)=0, \quad \lim _{t \rightarrow \infty} \hat{\mathbf{m}}^{\alpha \alpha^{\prime}}(\vec{q}, t)=0 .
$$

With the same argumentation as above we linearize Eqs. (30b) by taking $\hat{\mathbf{m}}^{\alpha \alpha^{\prime}}(\vec{q}, t)$ to be zero. Then, taking the time derivative of Eq. (30 b) and using (30a) we get for $\hat{\mathbf{S}}^{\alpha}(\vec{q}, t) \equiv\left(\mathbf{J}^{\alpha}(\vec{q})\right)^{-\frac{1}{2}} \mathbf{S}^{\alpha}(\vec{q}, t)$ :

$$
\ddot{\hat{\mathbf{S}}}^{\alpha}(\vec{q}, t)+\sum_{\alpha^{\prime}}\left(\hat{\mathbf{\Omega}}^{2}(\vec{q})\right)^{\alpha \alpha^{\prime}} \hat{\mathbf{S}}^{\alpha^{\prime}}(\vec{q}, t)=0
$$

with the frequency matrix squared:

$\hat{\mathbf{\Omega}}^{2}(\vec{q})^{\alpha \alpha^{\prime}}=\left(\mathbf{J}^{\alpha}(\vec{q})\right)^{\frac{1}{2}}\left[\mathbf{q}^{\alpha} \mathbf{S}^{-1}(\vec{q}) \mathbf{q}^{\alpha^{\prime}}-\mathbf{C}^{\alpha \alpha^{\prime}}(\vec{q})\right]\left(\mathbf{J}^{\alpha^{\prime}}(\vec{q})\right)^{\frac{1}{2}}$

Integration of (30a) from 0 to $t$ and substituting $\int_{0}^{t} d t^{\prime} \mathbf{S}^{\alpha^{\prime}}\left(\vec{q}, t^{\prime}\right)$ from Eqs. 30 ) with $\hat{\mathbf{m}}^{\alpha \alpha^{\prime}} \equiv 0$ allows to express $\hat{\mathbf{S}}(\vec{q}, t)$ by $\hat{\mathbf{S}}^{\alpha}(\vec{q}, t)$ :

$$
\begin{aligned}
\hat{\mathbf{S}}(\vec{q}, t)= & i \mathbf{F}(\vec{q}) \mathbf{S}^{-1}(\vec{q}) \sum_{\alpha, \alpha^{\prime}} \mathbf{q}^{\alpha}\left[\left(\mathbf{C}^{\beta \beta^{\prime}}(\vec{q})\right)^{-1}\right]^{\alpha \alpha^{\prime}} \\
& \times\left(\mathbf{J}^{\alpha^{\prime}}(\vec{q})\right)^{1 / 2} \hat{\mathbf{S}}^{\alpha^{\prime}}(\vec{q}, t)
\end{aligned}
$$

Similar to $\boldsymbol{\Omega}$, the matrix $\left(\hat{\boldsymbol{\Omega}}^{\alpha \alpha^{\prime}}\right)$ is non-diagonal in $l, l^{\prime}$ and in $\alpha, \alpha^{\prime}$. This again leads to a coupling between the translational and orientational modes, which are the eigenmodes of $\left(\hat{\boldsymbol{\Omega}}^{\alpha \alpha^{\prime}}\right)$. Now it is (see [6]):

$$
\mathbf{C}^{\alpha \alpha^{\prime}}(\vec{q})=\tilde{\mathbf{q}}^{\alpha} \tilde{\mathbf{C}}^{\alpha \alpha^{\prime}}(\vec{q}) \tilde{\mathbf{q}}^{\alpha^{\prime}}
$$


with:

$$
\lim _{\vec{q} \rightarrow 0} \tilde{C}_{l m, l^{\prime} m^{\prime}}^{\alpha \alpha^{\prime}}(\vec{q}) \neq 0
$$

and

$$
\begin{aligned}
\left(\tilde{\mathbf{q}}^{\alpha}\right)_{l m, l^{\prime} m^{\prime}}=\tilde{q}_{l m l^{\prime} m^{\prime}}^{\alpha} & =\tilde{q}_{l}^{\alpha} \delta_{l l^{\prime}} \delta_{m m^{\prime}} \\
\tilde{q}_{l}^{\alpha} & = \begin{cases}q, & (\alpha, l)=(T, 0) \\
1, & (\alpha, l) \neq(T, 0),\end{cases}
\end{aligned}
$$

not to be confused with $\mathbf{q}^{\alpha}$. Therefore, we get from Eq. (34) with Eqs. (6), (14), (36) and (38):

$$
\begin{array}{r}
\left(\hat{\mathbf{\Omega}}^{2}(\vec{q})\right)_{00,00}^{T T}=\frac{k_{B} T}{M} q^{2}\left(\mathbf{S}^{-1}(\vec{q})\right)_{00,00} \\
{\left[1+\left(\mathbf{S}(\vec{q}) \tilde{\mathbf{C}}^{T T}(\vec{q})\right)_{00,00}\right]}
\end{array}
$$

for the acoustic part of $\left(\left(\hat{\boldsymbol{\Omega}}^{2}\right)^{\alpha \alpha^{\prime}}\right)$. Taking into account that we have not normalized $\mathbf{C}^{\alpha \alpha^{\prime}}$ and $\tilde{\mathbf{C}}^{\alpha \alpha^{\prime}}$, the result Eq. (40) is completely analogous to the result derived in [57 for $(\hat{\boldsymbol{\Omega}}(\vec{q}))^{2}$ for simple one-component liquids. However, we note that in contrast to [57] the equations of motion for the rescaled correlators $\widehat{\hat{\mathbf{S}}}(\vec{q}, t)$ and $\hat{\mathbf{m}}^{\alpha \alpha^{\prime}}(\vec{q}, t)$ are not covariant, due to the splitting of the current density into a translational and rotational part. Therefore $\left(\hat{\boldsymbol{\Omega}}^{2}(\vec{q})\right)^{\alpha \alpha^{\prime}}$ is not a straight forward generalization of $\hat{\mathbf{\Omega}}^{2}(\vec{q})$ for simple liquids to molecular liquids.

The coupling between translational and orientational modes in the liquid and in the glass already on the linear level of the equations of motion is not surprising due to the interaction between translational and orientational degrees of freedom. But, it is also obvious that memory effects will lead to additional couplings and it is this point, which we will investigate in section III.

\section{Mode coupling theory}

In the preceding subsection we have neglected the memory kernels. Approaching the glass transition significant memory effects occur. Therefore $\mathbf{m}^{\alpha \alpha^{\prime}}$ must be taken into account. Using mode-coupling theory (MCT) an approximate expression for the slow part $\mathbf{m}^{\alpha \alpha^{\prime}}(\vec{q}, t)$ has been derived which leads to a closed set of equations for $\mathbf{S}(\vec{q}, t)$. This has been done for molecular systems using the molecular representation [5] and a site-site description 667. For a liquid of linear molecules we will use:

$$
\begin{aligned}
m_{l m, l^{\prime} m^{\prime}}^{\alpha \alpha^{\prime}}(\vec{q}, t) & \approx \frac{\sqrt{I_{\alpha} I_{\alpha^{\prime}}}}{k_{B} T} \Gamma_{l m, l^{\prime} m^{\prime}}^{\alpha \alpha^{\prime}}(\vec{q}) \delta(t)+ \\
& +\left(m_{l m, l^{\prime} m^{\prime}}^{\alpha \alpha^{\prime}}(\vec{q}, t)\right)_{\text {slow }}
\end{aligned}
$$

where the first and second term on the r.h.s. of Eq. (41) accounts for the fast and the slow contributions, respectively. MCT yields [6]

$$
\begin{array}{r}
\left(m_{l m, l^{\prime} m^{\prime}}^{\alpha \alpha^{\prime}}(\vec{q}, t)\right)_{\text {slow }}=\frac{1}{2 N} \sum_{\substack{\vec{q}_{1} \vec{q}_{2} \\
\vec{q}_{1}+\vec{q}_{2}=\vec{q}}} \sum_{\substack{l_{1} m_{1} \\
l_{2} m_{2}}} \sum_{l_{1}^{\prime} m_{1}^{\prime}} \times \\
\quad \times V_{2}^{\prime} m_{2}^{\prime} \\
\quad \alpha_{l m, l^{\prime} m^{\prime}}^{\prime}\left(\vec{q} \mid \vec{q}_{1} l_{1} m_{1}, l_{1}^{\prime} m_{1}^{\prime} ; \vec{q}_{2} l_{2} m_{2}, l_{2}^{\prime} m_{2}^{\prime}\right) \\
\times S_{l_{1} m_{1}, l_{1}^{\prime} m_{1}^{\prime}}\left(\vec{q}_{1}, t\right) S_{l_{2} m_{2}, l_{2}^{\prime} m_{2}^{\prime}}\left(\vec{q}_{2}, t\right)
\end{array}
$$

The explicit expressions for the vertices $\mathbf{V}$ are given in Ref. [6]. Equations (10) together with equations (41) and (43) are a closed set of equations for $S_{l m, l^{\prime} m^{\prime}}(\vec{q}, t)$ which need the damping coefficients $\Gamma_{l m, l^{\prime} m^{\prime}}^{\alpha \alpha^{\prime}}(\vec{q})$ and the static correlators $S_{l m, l^{\prime} m^{\prime}}(\vec{q})$ as an input. The latter uniquely determine the vertices.

It is obvious that the MCT-polynomial $\left(m_{l m, l^{\prime} m^{\prime}}^{\alpha \alpha^{\prime}}\right)_{\text {slow }}$ leads to additional coupling between the correlators $S_{l m, l^{\prime} m^{\prime}}(\vec{q}, t)$. Due to this nonlinearity the MCTequations (10), (41) and (43) can only be solved numerically. We have performed such a numerical solution for dipolar hard spheres, one of the simplest systems involving translational and rotational motion.

\section{Dipolar hard sphere liquid}

The investigation of dipolar hard spheres (DHS) has the advantage that approximate analytical expressions [70 for the static correlators are known.

We consider a system of $\mathrm{N}$ hard spheres with homogeneous number density $\rho$, diameter $d$, mass $M$, moment of inertia $I=\frac{1}{10} M d^{2}$ and dipolar moment $\mu$. The origin of the body fixed frame is chosen to coincide with the center of mass of each sphere, which is the natural choice. Since the density of the particles is homogeneous, the center of mass is equal to the center of the spheres. The reader should note that the MCT-equations (10), (41) and (43) and even the original exact equations of motion Eqs. (10) are not covariant (i.e. invariant in their form) under a shift of the reference point for the body-fixed frame. In order to get covariant equations one has to project on the individual Cartesian components of $\vec{j}_{l m}^{T}(\vec{q})$ and $\vec{j}_{l m}^{R}(\vec{q})$. Nevertheless, we think that the present equations are a reasonable good approach to the dynamics of molecular liquids, because of the natural choice of the reference frame. Of course, this point needs additional investigations. The advantage of MCT in site-site representation [67] is that this problem does not occur, because no reference point must be chosen.

The physical control parameters are the packing fraction $\phi=\frac{\pi}{6} \rho d^{3}$ and the temperature $T$. In the following the length unit is chosen such that $d=1$. In addition we choose $M=1$ and $\mu=1$. This choice means that time $t$ and temperature $T$ are measured in units of $M^{1 / 2} d^{5 / 2} / \mu$ and $k_{B}^{-1} \mu^{2} / d^{3}$, respectively. In the following we will use $T^{*}=T /\left(\mu^{2} / k_{B} d^{3}\right)$ as dimensionless temperature. 
As already stated, the MCT-equations (10), 41 and (43) require $\Gamma_{l m, l^{\prime} m^{\prime}}^{\alpha \alpha^{\prime}}(\vec{q})$ and $S_{l m, l^{\prime} m^{\prime}}(\vec{q})$ as an input. Throughout this paper we will put all damping coefficients $\Gamma_{l m, l^{\prime} m^{\prime}}^{\alpha \alpha^{\prime}}$ to zero. The static correlators $S_{l m, l^{\prime} m^{\prime}}(\vec{q})$ are obtained from Wertheim's solution who used PercusYevick and mean spherical approximation [70] This leads to

$$
S_{l m, l^{\prime} m^{\prime}}(\vec{q}) \approx \begin{cases}S_{l m}(q) \delta_{l l^{\prime}} \delta_{m m^{\prime}} & , \quad l=0,1, l^{\prime}=0,1 \\ \delta_{l l^{\prime}} \delta_{m m^{\prime}} & , \quad \text { otherwise }\end{cases}
$$

The vertices of the memory kernels are bilinear in the direct correlation functions

$$
c_{l m}(q)=\frac{4 \pi}{\rho}\left[1-\frac{1}{S_{l m}(q)}\right]
$$

Since we have chosen the so-called $q$-frame [71] (for which $\left.\vec{q}=\vec{q}_{0} \equiv(0,0, q)\right)$ the correlators and the kernels become diagonal in $m$ and $m^{\prime}$ [6]. The fact that the static correlators are diagonal in $l$ and $l^{\prime}$ and are structureless for $l, l^{\prime} \geq 2$ is an artefact of both approximations. Additional shortcomings are (i) the independence of the center of mass correlator $S_{00}(q)$ on temperature and (ii) the smooth behavior of $S_{l m}(q)$ at $\phi_{\mathrm{rcp}} \simeq 0.64$, the value for random close packing. The static correlators from Wertheim's approach exhibit a divergency at $\phi=\phi_{\max }=1$, only. We will come back to these points below.

Due to (46) and (47) only mode coupling occurs between the time dependent correlators with $l_{1}, l_{1}^{\prime}, l_{2}, l_{2}^{\prime}$ smaller or equal to one. In order to simplify the MCTequations as much as possible we use the additional approximations (in the $q$-frame)

$$
S_{l m, l^{\prime} m^{\prime}}(\vec{q}, t) \approx \begin{cases}S_{l m}(q, t) \delta_{l l^{\prime}} \delta_{m m^{\prime}} & , \quad l=0,1, l^{\prime}=0,1 \\ 0 & , \quad \text { otherwise }\end{cases}
$$

$m_{l m, l^{\prime} m^{\prime}}^{\alpha \alpha^{\prime}}(\vec{q}, t) \approx \begin{cases}m_{l m}^{\alpha \alpha^{\prime}}(q, t) \delta_{l l^{\prime}} \delta_{m m^{\prime}} & , \quad l=0,1, l^{\prime}=0,1 \\ 0 & , \quad \text { otherwise }\end{cases}$

In section IIB we have shown that translational and orientational dynamics is already coupled on the linear level due to non-diagonality in $l$ and $l^{\prime}$. Above we have chosen all correlators to be diagonal in $l$ and $l^{\prime}$. Therefore, a coupling between $S_{00}(q, t)$ and $S_{l m}(q, t)$ with $l>0$ can only originate from mode-coupling effects. Therefore, our diagonalization approximation (which is even exact in our case due to the restriction of $l$ and $l^{\prime}$ to 0 and 1) allows to study the influence of the mode-coupling terms on the microscopic dynamics without interfering with the direct coupling mechanism between the correlators $S_{l m}(q, t)$ discussed in section IIB.
The memory kernels (cf. Eq. (43)) in the $q$-frame $m_{l m, l^{\prime} m^{\prime}}^{\alpha \alpha^{\prime}}(\vec{q}, t)=m_{l m}^{\alpha \alpha^{\prime}}(q, t) \delta_{l l^{\prime}} \delta_{m m^{\prime}}$ contain the following couplings [6]:

$m_{00}^{\alpha \alpha^{\prime}}(q, t) \leftrightarrow S_{00}\left(q_{1}, t\right) S_{00}\left(q_{2}, t\right)$ and $S_{1 m_{1}}\left(q_{1}, t\right) S_{1 m_{2}}\left(q_{2}, t\right)$

$m_{1 m}^{\alpha \alpha^{\prime}}(q, t) \leftrightarrow S_{00}\left(q_{1}, t\right) S_{1 m_{2}}\left(q_{2}, t\right)$ and $S_{1 m_{1}}\left(q_{1}, t\right) S_{00}\left(q_{2}, t\right)$

Eq. (50) shows that the center of mass correlator $S_{00}(q, t)$ may undergo a glass transition independently from the dipoles whereas Eq. (51) demonstrates that the dipoles are "slaved" by the center of mass dynamics and can freeze only if $S_{00}(q, t)$ has become non-ergodic. In order that $S_{00}(q, t)$ and $S_{1 m}(q, t)$ freeze simultaneously the vertices of the bilinear terms in Eq. (51) must be large enough. This happens at low enough temperatures. Since $S_{l,-m}(q, t) \equiv S_{l, m}(q, t)$ [6], there are three independent correlators which are the center of mass correlation function $S_{00}(q, t)$ and two dipolar ones $S_{1 m}(q, t)$, $m=0,1$. In the following we consider the normalized correlators $\phi_{l m}(q, t)=S_{l m}(q, t) / S_{l m}(q)$.

The three static correlators are shown in Fig. 1 for two different pairs of $\left(\phi, T^{*}\right)$. Three main features can be seen. First, the $q$-variation of $S_{00}$ of the "longitudinal " dipolar correlator and $S_{10}$ is rather similar to each other and resembles that of a simple liquid with a well pronounced main peak at $q_{\max } \approx 2 \pi / \bar{a}$, where $\bar{a} \approx d=1$, the mean distance between nearest neighbors. The "transversal" dipolar correlator $S_{11}(q)$ behaves quite differently. It exhibits only one peak which is at $q=0$ and it becomes almost structureless for $q>\Delta q$, the peak width at half maximum. Second, $S_{11}(q=0)$ increases with decreasing temperature, whereas $\triangle q$ decreases. This behavior signals a medium range orientational order due to a precursor of ferrofluid order. This order is induced by an enhancement of the dipolar interactions at lower temperatures with respect to the hard core repulsion. Third, $S_{1 m}(q=0)$ depends on $m$, because of the long range nature of the dipolar interactions.

Besides the truncation at $l=1$ we must also truncate and discretize the $q$-variable. Since the reduction (for $N \rightarrow \infty$ ) to a single integral of the sum over $\vec{q}_{1}$ and $\overrightarrow{q_{2}}$ in Eq. (43) for simple liquids [72] cannot easily be used for molecular liquids the number of steps to calculate this sum increases quadratically with the number of $q$-values, instead of a linear increase for simple liquids. This fact makes the numerical solution of the molecular MCT-equations rather CPU-time consuming. Therefore we decided to choose a non-equidistant distribution of 30 $q$-values between $q_{\text {low }} \simeq 0.51$ and $q_{u p} \simeq 40$. These values were generated by the non-linear relation

$$
q_{\nu}=\frac{1}{\alpha} \operatorname{arctanh}\left(\nu \Delta_{x}\right), \quad \nu=1,2, \ldots, 30 .
$$


with $\Delta_{x}=\frac{\tanh \left(\alpha q_{c o}\right)}{31}$ and $q_{c o}=50$. The parameter $\alpha$ has been chosen such that the main peak of, e.g. $S_{00}(q)$, is still in the linear regime of arctanh. The right of Fig. 11 demonstrates this for $\alpha=0.065$. It is obvious from (52) that for $q<\alpha^{-1}$ the $q_{\nu}$ are almost equidistant and for $q>\alpha^{-1}$ they become more and more diluted. Since the large $q$-regime is not as important as the range around the main peak in $S_{00}(q)$ or that at $q=0$ in $S_{11}(q)$ (which drive the glass transition) our choice for $q_{\nu}$ should not influence our results, at least qualitatively. We have solved the molecular MCT-equations (10), (41) and (43) in time space using an algorithm already developed to solve the MCT-equations for simple liquids 73. However, our numerical procedure differs from that by Götze et al. [74 for the single dumbbell in an isotropic liquid. These authors introduced an effective memory kernel (independent on $\alpha$ and $\alpha^{\prime}$ ) and an effective microscopic frequency.

Before we come to the dynamics let us shortly discuss the phase diagram for a glass transition which was already calculated 6,75]. Throughout the rest of this paper all the results are given in the $q$-frame. The modification with respect to Refs. 66.75 of the $q$-discretization leads to a small quantitative change of the glass transition lines, but without changing the topology. Therefore we have calculated again non-ergodicity parameters

$$
f_{l m}(q)=\lim _{t \rightarrow \infty} \phi_{l m}(q, t)
$$

as a function of $\phi$ and $T^{*}$ from which the phase diagram is obtained (Figure 2). Since $\phi$ can not exceed $\phi_{r c p} \simeq 0.64$, the value for random close packing of hard spheres, we have plotted only $\phi \leq \phi_{r c p}$. There are two significant temperatures $T_{1}^{*}$ and $T_{2}^{*}$. At $T_{1}^{*}$ the critical line $\phi_{\text {type A }}^{c}\left(T^{*}\right)$ (dashed line in Figure 2) reaches $\phi=\phi_{r p c}$ and at $T_{2}^{*}$ it merges into the critical line $\phi_{\text {type } \mathrm{B}}^{c}\left(T^{*}\right)$ (solid line in Figure 2). These two temperatures have the following meaning. For $T^{*}>T_{1}^{*}$ an increase of $\phi$ leads at $\phi_{\text {type B }}^{c}\left(T^{*}\right)$ to a glass transition for the center of mass motion, but not for the dipoles. Choosing $T^{*}$ between $T_{1}^{*}$ and $T_{2}^{*}$ again a glass transition of the center of mass motion occurs at $\phi_{\text {type B }}^{c}\left(T^{*}\right)$. But increasing $\phi$ beyond $\phi_{\text {type } \mathrm{B}}^{c}\left(T^{*}\right)$ a spin glass like transition for the dipoles occurs at $\phi_{\text {type A }}^{c}\left(T^{*}\right)$. Below $T_{2}^{*}$, center of mass and dipolar dynamics freeze simultaneously at $\phi_{\text {type } \mathrm{B}}^{c}\left(T^{*}\right)$. For $T^{*}>T_{2}^{*}$ the critical value $\phi_{\text {type } \mathrm{B}}^{c}\left(T^{*}\right)$ is identical to that for hard spheres $\phi_{H S}^{c}$. For the present choice of $q$ discretization we find $\phi_{H S}^{c} \simeq 0.5265$ which does not differ much from the more precise value $\phi_{H S}^{c} \simeq 0.516$ obtained with 100 equidistant $q$-values [76].

The glass transition at high and low temperatures is driven by different physical mechanisms. At high temperatures it is the so-called cage effect, which leads to the freezing of the liquid into a non-ergodic phase, due to an increase with increasing density of the main peak of the center of mass correlator $S_{00}(q)$. Lowering the temperature enhances the role of the dipolar interactions which leads to a strong increase of the peak at $q=0$ of the "transversal" dipolar correlator $S_{11}(q)$. The increase of the dipolar correlations are accompanied by a decrease of the center of mass correlations (cf. Fig. 11), and take over the role of the cage effect. This behavior reflects itself in the non-ergodicity parameters. $f_{11}(q=0)$ increases with decreasing temperature much stronger than $f_{00}\left(q_{\max }\right)$. For details the reader is referred to [6].

The properties of the phase diagram require some more comments: (i) the $T^{*}$-independence of $\phi_{\text {type B }}^{c}\left(T^{*}\right)$ for $T^{*}>T_{1}^{*}$ originates from the $T^{*}$-independence of $S_{00}(q)$, which is an artefact of Wertheim's solution. Removing this artefact will result in a $T^{*}$-dependence of $\phi_{\text {type B }}^{c}(T)$ for all $T^{*}$ with $\lim _{T^{*} \rightarrow \infty} \phi_{\text {type B }}^{c}\left(T^{*}\right)=\phi_{H S}^{c}$, since dipolar interactions will be irrelevant at infinite temperature (ii), the existence of $T_{1}^{*}$ relies on the fact that $\phi_{\text {type A }}^{c}\left(T^{*}\right)$ approaches the value $\phi_{\text {rcp }}$ at a finite temperature. This is true when the static correlators within Percus-Yevick approximation are used. Whether or not an improved theory reproducing the singular behavior at $\phi_{\text {rcp }}$ would lead to the same conclusion is not obvious. Since such a theory does not exist, we can not exclude that $\phi_{\text {type A }}^{c}\left(T^{*}\right)$ only approaches $\phi_{\text {rcp }}$ at $T^{*}=\infty$.

Now we turn to the time or frequency dependent features of dipolar hard spheres. We have studied the control parameter dependence of the dynamics along the paths A, B, C and D, indicated in Figure 2. In contrast to the determination of the long time behavior inertia effects will play an important role in the microscopic time or frequency regime. In the liquid, these inertia effects enter through the microscopic frequency matrix $\boldsymbol{\Omega}(\vec{q})$. Due to the diagonality of $\mathbf{S}(\vec{q})$ the matrix $\boldsymbol{\Omega}(\vec{q})$ becomes diagonal with diagonal elements (in $q$-frame) which follows from Eq. (20):

$$
\Omega_{l m}(q)=\sqrt{\left[\frac{k_{B} T}{M} q^{2}+\frac{k_{B} T}{I} l(l+1)\right] / S_{l m}(q)},
$$

restricted to $l=0,1$. Figure 3 depicts $\Omega_{l m}(q)$ for two different pairs $\left(\phi, T^{*}\right)$ in vicinity of the critical line $\phi_{\text {type B }}^{c}\left(T^{*}\right)$. This Figure reveals two features which stem from the properties of $S_{l m}(q)$ discussed above. First, $\Omega_{1 m}(q=0)$ depends on $m$. Second, and most important, a "softening" of the "optic" frequency $\Omega_{11}(q)$ with $q \rightarrow 0$ is observed for decreasing temperature $T^{*}$ for which the role of dipolar interactions (compared to the hard core repulsion) becomes more and more enhanced. This "softening" comes from the strong increase of $S_{11}(q)$ for $q \rightarrow 0$ (see Fig. 11) and has its physical origin in the occurrence of a medium range orientational order of the dipoles. The inverse width $(\Delta q)^{-1}$ of the peak in $S_{11}(q)$ at $q=0$ is a measure for the length scale for this orientational order. $(\Delta q)^{-1}$ increases with decreasing temperature (see Fig. 1). We will come back to this point in the final section.

The microscopic frequencies in the glass phase are given by the "renormalized" frequency matrix $\left(\left(\hat{\Omega}^{2}(\vec{q})\right)^{\alpha \alpha^{\prime}}\right)$. Taking again into account the diago- 
nalization with respect to $l$ and $l^{\prime}$ we obtain from Eq. (34) with Eqs. (14) and (36)

$$
\begin{aligned}
\left(\hat{\mathbf{\Omega}}_{l m}^{2}(q)\right)^{\alpha \alpha^{\prime}} & =\frac{k_{B} T}{\left(I_{\alpha} I_{\alpha}^{\prime}\right)^{1 / 2}} \frac{1}{S_{l m}(q)} \times \\
& \times\left[q_{l}^{\alpha} q_{l}^{\alpha^{\prime}}+\tilde{q}_{l}^{\alpha} \tilde{q}_{l}^{\alpha^{\prime}} \tilde{C}_{l m}^{\alpha \alpha^{\prime}}(q) S_{l m}(q)\right]
\end{aligned}
$$

Since $\tilde{C}_{00}^{\alpha \alpha^{\prime}}(q)$ is different from zero for $\alpha=\alpha^{\prime}=T$, only, we get with Eqs. (6), (27), (36) and (38):

$$
\hat{\boldsymbol{\Omega}}_{00}(q)=\Omega_{00}(q) /\left(1-f_{00}(q)\right)^{1 / 2} .
$$

$\tilde{C}_{1 m}^{\alpha \alpha^{\prime}}(q)$ is nonzero for all $\left(\alpha, \alpha^{\prime}\right)$. Therefore two eigenfrequencies $\hat{\Omega}_{1 m}^{ \pm}(q)$ exist for each $m . \hat{\mathbf{\Omega}}_{00}(q)$ and $\hat{\Omega}_{1 m}^{ \pm}(q)$ are shown in Fig. 4. the "renormalized" frequencies require the non-ergodicity parameters as an input. The latter become less accurate for small $q$, because the discretization of the $q$-values influences the results much stronger for decreasing $q$. Therefore we do not present data below $q=1$. Of course it is $\hat{\Omega}_{00}(q) \rightarrow \hat{c} q$ and $\hat{\Omega}_{1 m}^{ \pm}(q) \rightarrow \hat{\Omega}_{1 m}^{ \pm}(0)>0$ for $q \rightarrow 0$. Note that (i) $\hat{\Omega}_{11}^{ \pm}(q)$ does not vary much with $q$ and (ii) that $\hat{\Omega}_{11}^{-}(q) \ll \hat{\Omega}_{00}\left(q_{\max } \simeq 7\right)$.

Now let us turn to the equations (10) including the time-dependent memory kernels given by (41) and 43. From the solutions of these MCT-equations we get the normalized correlators $\phi_{l m}(q, t)$ or equivalently the corresponding susceptibility spectra $\chi_{l m}^{\prime \prime}(q, \omega)$ related to the correlation spectra $\phi_{l m}^{\prime \prime}(q, \omega)$ by $\chi_{l m}^{\prime \prime}(q, \omega)=\omega \phi_{l m}^{\prime \prime}(q, \omega)$. Figures 5 and 6 show $\phi_{l m}(q, t)$ and $\chi_{l m}^{\prime \prime}(q, \omega)$ for $q \simeq 4.7$ along path $\mathrm{A}$ in Figure 2. Figure 5 clearly demonstrates for all correlators the formation of a plateau and the tremendous slowing down of the relaxation with increasing $\phi$ which stops at $\phi_{c}\left(T^{*}\right) \simeq 0.3782$.

The dynamical features are better recognized in $\omega$ space. Before we discuss these results, let us comment on the quality of our $\omega$-dependent data. Because the glassy dynamics involves many decades in time one has to use a special algorithm, which decimates the time step with increasing time, i.e. one chooses an initial time increment $h$ to discretize the time axis [73]. After a certain number of time steps the increment is doubled, etc. Although the $t$-dependent data shown in Fig. 5 look continuous, they exhibit tiny discontinuities of the correlators and of their first time derivative at those times at which the increment is doubled. These discontinuities which could be diminished by an increase of the CPU-time lead to the wiggles which can, e.g. be seen in Fig. 6. However, we have checked by variation of the parameters $h$, the boundary values of the Fourier integrals, etc. that the features we will address below are not influenced by the wiggles. Further, the low frequency wing of the $\alpha$-peak for $\chi_{l m}^{\prime \prime}(q, \omega)$ in Fig. 6 shows a rather small deviation from a linear $\omega$-dependence, as it should be. This failure is due to the choice of the lower bound for the Fourier integral. If this is taken to be zero, the wiggles become more pronounced. Therefore, choosing a nonzero bound is a compromise between the reduction of the wiggles and a small deviation from the linearity in $\omega$ for $\omega \rightarrow 0$. We have also used a spline-technique for smoothing the data in time space. The Fourier transform of those has reproduced all the relevant features obtained from the original $t$-dependent correlators.

Let us now discuss the spectra. For $\phi<\phi_{c}\left(T_{A}^{*}\right)$, i.e. in the liquid phase, Figure 6 left column reveals the existence of three peaks for $\chi_{00}^{\prime \prime}(q \simeq 4.7, \omega)$ and two peaks for $\chi_{11}^{\prime \prime}(q \simeq 4.7, \omega)$ and $\chi_{10}^{\prime \prime}(q \simeq 4.7, \omega)$. The low frequency peak in all spectra $\chi_{l m}^{\prime \prime}(q \simeq 4.7, \omega)$ is the $\alpha$-peak related to the slowing down of the structural relaxation (translational and rotational degrees of freedom). $\chi_{00}^{\prime \prime}(q \simeq 4.7, \omega)$ has a high-frequency (hf) peak at $\omega_{h f} \approx 10$ and an additional peak at $\omega_{o p} \simeq 1$, i.e. about one decade below the hf-peak. $\quad \chi_{10}^{\prime \prime}(q \simeq 4.7, \omega)$ and $\chi_{11}^{\prime \prime}(q \simeq 4.7, \omega)$ exhibits only one peak in the microscopic frequency domain at $\omega \approx \omega_{o p}$. Below we will show that the peak in $\chi_{00}^{\prime \prime}(q \simeq 4.7, \omega)$ at $\omega \approx \omega_{o p}$ originates from the orientational dynamics. Therefore we call it "orientational" peak (op).

The situation for $\phi>\phi_{c}\left(T_{A}^{*}\right)$, i.e. in the glass phase, is similar with two exceptions (cf. right column of Fig. 6). First, it is clear that there is no $\alpha$-peak anymore and second an additional peak located between the op and the hf-peak exists in $\chi_{00}^{\prime \prime}(q \simeq 4.7, \omega)$ for $\phi=0.53$ and 0.60 , i.e. deep in the glass phase. Even a further "peak" looking more like a shoulder appears for $\phi=0.6$. Such additional peaks can also be seen in $\chi_{10}^{\prime \prime}(q \simeq 4.7, \omega)$ and $\chi_{11}^{\prime \prime}(q \simeq 4.7, \omega)$ (cf. right column of Fig. 6)

In the following we will concentrate ourselves onto the microscopic regime. Therefore we can use a linear $\omega$-scale, which allows us to recognize the $\omega$ - and $\phi$ dependence (which we have described above) much better for $0.1<\omega<10$. This is done in Figure 7 for the data from Figure 6. Both microscopic peaks at $\omega_{o p} \approx 1$ and $\omega_{h f} \approx 10$ can clearly be seen in the compressibility spectrum $\chi_{00}^{\prime \prime}(q \simeq 4.7, \omega)$. Whereas the intensity of the hf-peak increases with increasing $\phi$, the op becomes more pronounced when approaching the glass transition from the liquid side. In the glass phase at $\phi=0.44$ and $\phi=0.48$ it is less prominent but again becomes more pronounced for $\phi>0.5$, although its intensity decreases. The appearance of the intermediate peak for $\phi=0.53$ and $\phi=0.60$ at $\omega \approx 6$ and $\omega \approx 8$, respectively, can be observed as well as the shoulder at $\omega \approx 4$. Due to the linear $\omega$-scale we clearly see that $\chi_{10}^{\prime \prime}(q \simeq 4.7, \omega)$ also exhibits an intermediate peak for $\phi=0.53$ and $\phi=0.60$, i. e. $\chi_{10}^{\prime \prime}(q \simeq 4.7, \omega)$ resembles $\chi_{00}^{\prime \prime}(q \simeq 4.7, \omega)$ but with the opposite $\phi$-dependence of the intensity of the op and hf-peak for $\phi>\phi_{c}\left(T_{A}^{*}\right) \cdot \chi_{11}^{\prime \prime}(q \simeq 4.7, \omega)$ which is proportional to the dielectric loss $\epsilon^{\prime \prime}(q \simeq 4.7, \omega)$, possesses only one well pronounced peak at $\omega \approx \omega_{o p}$. To check how far the features depend on the path through a critical point, we have investigated $\chi_{l m}^{\prime \prime}(q \simeq 4.7, \omega)$ along path $\mathrm{C}$ (see Figure 1) for which $\phi=\phi_{c}=0.3786$ is fixed. This allows 
to study how far density and temperature variation lead to similar or different susceptibility spectra. The results for path $\mathrm{C}$ are given in Figure 8 . $\chi_{00}^{\prime \prime}(q \simeq 4.7, \omega)$ shows an op and a hf-peak at $\omega_{o p} \approx 1$ and $\omega_{h f} \approx 10$, respectively. Their corresponding intensities behave qualitatively similar for decreasing $T^{*}$ as we have found along path $\mathrm{A}$ for increasing $\phi$. Intermediate peaks between op and hf-peak do not occur. $\chi_{10}^{\prime \prime}(q \simeq 4.7, \omega)$ differs from the result along path $\mathrm{A}$, whereas $\chi_{11}^{\prime \prime}(q \simeq 4.7, \omega)$ looks similar.

Translational and orientational dynamics freeze on path $\mathrm{A}$ and $\mathrm{C}$ simultaneously. Therefore we have also calculated $\chi_{l m}^{\prime \prime}(q \simeq 4.7, \omega)$ on path B for fixed $T^{*}=$ 0.30 where $\phi_{00}(q, t)$ freezes at $\phi_{c}^{H S} \simeq 0.5265$ first, whereas $\phi_{1 m}(q, t)$ undergoes a spin-glass-like transition at $\phi_{\text {type } \mathrm{A}}^{c}\left(T^{*}=0.3\right) \simeq 0.62$. The corresponding results are presented in Figure 9. $\chi_{00}^{\prime \prime}(q \simeq 4.7, \omega)$ and $\chi_{1 m}^{\prime \prime}(q \simeq$ $4.7, \omega)$ exhibit a well pronounced main peak. Its position depends sensitively on $\phi$ in case of $\chi_{00}^{\prime \prime}(q \simeq 4.7, \omega)$ and is practically $\phi$ independent for $\chi_{1 m}^{\prime \prime}(q \simeq 4.7, \omega)$. The latter quantity also pocesses a small peak at $\omega \approx 10$, which originates from the translational motion via translationrotation-coupling.

A peak in the susceptibility spectra can be either of relaxational or oscillatory origin. To distinguish between both types of behavior one must study the $\omega$-dependence of the correlation spectra $\phi_{l m}^{\prime \prime}(q, \omega)$. Spectra from neutron scattering are superpositions of $\phi_{l m}^{\prime \prime}(q, \omega)$ [65]. A peak in $\phi_{l m}^{\prime \prime}(q, \omega)$ at $\omega>0$ proves the existence of an oscillation whereas a peak at $\omega=0$ is of purely relaxational type. The corresponding peak width is a measure of the damping. These correlation spectra are shown in Figure 10 and 11 for path A and path B, respectively. For $\phi<\phi_{c}\left(T^{*}\right)$ (not shown in Fig. 10 and 11) there is no peak at nonzero frequency, except for the hf-peak. Therefore the op we have found in $\chi_{l m}^{\prime \prime}(q \simeq 4.7, \omega)$ in the liquid phase is a purely relaxational excitation. Now let us discuss the correlation spectra in the glass phase. Figure 10 shows that in the glass, but close to the critical packing fraction, there is a hf-peak at $\hat{\omega}_{h f} \approx 10$. Deeper in the glass the position of that hf-peak shifts to higher frequencies and an op at $\hat{\omega}_{o p} \approx 1$ appears. Decreasing $\phi$ even more an intermediate peak between the op and the hf-peak is produced as well. The position of the op and intermediate peak shifts to higher frequencies with decreasing $\phi$ similar to the hf-peak, due to an increase of the glass-stiffness. The $\phi$-dependence of $\phi_{l m}^{\prime \prime}(q \simeq 4.7, \omega)$ at much higher temperature $T^{*}=0.3$ (path B in Figure 1) is presented in Figure 11. We observe a similar behavior as in Figure 10, i.e. besides the hf-peak at $\hat{\omega}_{h f}=20-60$ for $0.56 \leq \phi \leq 0.63$ there occurs the op at $\hat{\omega}_{o p} \approx 1$ and for $\phi=0.60$ and $\phi=0.63$ a shoulder at about $\omega \approx 20$ which corresponds to the intermediate peak in Figure 10 .

One of the conclusions we can draw from $\chi_{l m}^{\prime \prime}(q \simeq$ $4.7, \omega)$ and $\phi_{l m}^{\prime \prime}(q \simeq 4.7, \omega)$ is that there is an op at $\omega_{o p} \approx \hat{\omega}_{o p} \approx 1$ roughly one decade below a hf-peak. The hf-peak relates to a damped oscillation for the investigated range of $T^{*}$ and $\phi$, whereas the op changes its char- acter from purely relaxational to damped oscillational behavior under going from the liquid to the glass. Since the dipolar spectra $\chi_{1 m}^{\prime \prime}(q \simeq 4.7, \omega)$ and $\phi_{1 m}^{\prime \prime}(q \simeq 4.7, \omega)$ have a main peak at about $\omega \approx 1$ it is tempting to associate the op in $\chi_{00}^{\prime \prime}(q \simeq 4.7, \omega)$ and $\phi_{00}^{\prime \prime}(q \simeq 4.7, \omega)$ with the orientational degrees of freedom and their coupling (via mode coupling effects) to the translational ones. If this interpretation is correct then the op must exhibit an isotope effect with respect to a change of the moment of inertia $I$. Figure 12 presents $\chi_{l m}^{\prime \prime}(q \simeq 4.7, \omega)$ at $T_{A}^{*}=0.04, \phi=0.381$ for $I=\frac{1}{10}$ (the correct value for a hard sphere with $\mathrm{M}=1$ and $\mathrm{d}=1$ and homogeneous mass distribution), 1 and 10 . From this result we find

$$
\omega_{o p} \propto \frac{1}{\sqrt{I}} .
$$

This type of isotope effect also occurs for $\phi_{l m}^{\prime \prime}(q, \omega)$ which is shown in Figure 13 for $\phi_{l m}^{\prime \prime}(q \simeq 4.7, \omega)$ at $T_{A}^{*}=0.04$, $\phi=0.530$ and for $I=\frac{1}{10}, 1$ and 10 . This result yields:

$$
\hat{\omega}_{o p} \propto \frac{1}{\sqrt{I}} .
$$

The approximate scaling of $\omega_{o p}$ and $\hat{\omega}_{o p}$ with $1 / \sqrt{I}$ strongly supports the orientational origin of the op. Since $\omega_{h f}$ and $\hat{\omega}_{h f}$ are rather insensitive on a change of $I$ their origin must lie in the translational motion.

So far we have not studied the $q$-dependence of all of these peaks. Since this may elucidate more the features of the several peaks, we present $\chi_{l m}^{\prime \prime}(q, \omega)$ at $T_{A}^{*}=0.04$, $\phi=0.381$ in the glass but near the glass transition and $\phi_{l m}^{\prime \prime}(q, \omega)$ at $T_{A}^{*}=0.04, \phi=0.53$ deeper in the glass in Figures 14 and 15, respectively. The result for $\chi_{00}^{\prime \prime}(q, \omega)$ shows a nearly $q$-independent position of the op at $\omega_{o p} \approx$ 1 for $1.0 \leq q \leq 10.6$, whereas the position $\omega_{h f}$ of the hf-peak changes with $q$. A similar $q$-sensitivity holds for the position of the main microscopic peak in $\chi_{1 m}^{\prime \prime}(q, \omega)$ for $m=0$ and 1 . The result for $\phi_{l m}^{\prime \prime}(q, \omega)$ yields the same $q$ independence of the op at $\hat{\omega}_{o p} \simeq 1$ and a high sensitivity of the position of the hf-peak at $\hat{\omega}_{h f} \approx 10$. The high frequency peak is practically absent within a "window" around $q=6.5$ and it appears below and above that window.

Finally, let us comment on the dependence of the peaks on $T^{*}$ and $\phi$ as well as on $\alpha$, which characterizes the distribution of the values for $q$ (cf. Eq. (52) and Figure 11). Let us start with the $\alpha$-dependence. Figure 16 presents $\chi_{00}^{\prime \prime}\left(\bar{q}_{\max }, \omega\right)$ at $T_{A}^{*}=0.04, \phi=0.381$ for three different values of $\alpha . \bar{q}_{\max }$ which depends on $\alpha$ has been chosen as that $q$-value closest to the main maximum of $S_{00}(q)$. Although the peak positions and also the intensity varies with $\alpha$ the qualitative features do not depend on $\alpha$, at least for reasonable chosen values for $\alpha$.

The $T^{*}$-dependence of the position $\omega_{o p}$ and of the height $h_{o p}$ of the op for $q \simeq 4.7$ is shown in Figure 17 a for $\phi_{D}=0.525$ along path $\mathrm{D}$. The corresponding $\phi$ dependence for the same $q$ value and $T_{A}^{*}=0.04$ along 
path $\mathrm{A}$ is given in Figure 17b. $h_{o p}$ follows a linear $T^{*}$ dependence between $T^{*}=0.04$ (the lowest temperature we have studied) and $T^{*} \approx 0.1$. In a temperature region where $\omega_{o p}$ has a minimum, $h_{o p}$ shows a crossover to a constant. As can be observed from Figure $17 \mathrm{~b}$ the position $\omega_{o p}$ and the height $h_{o p}$ are almost constant below $\phi_{c}\left(T_{A}^{*}=0.04\right) \simeq 0.3782$ and increase with $\phi$ above that value.

\section{SCHEMATIC MODEL}

In this section we will demonstrate that besides a high frequency peak the occurrence of an additional microscopic low frequency peak can be obtained from a schematic model. For dipolar hard spheres it has been shown in section II that this additional peak in $\chi_{00}^{\prime \prime}(q, \omega)$ and $\phi_{00}^{\prime \prime}(q, \omega)$ has its origin in the orientational degrees of freedom and their coupling to the center of mass motion. Therefore the schematic model must contain at least two correlators $\phi_{0}(t)$ and $\phi_{1}(t)$, describing center of mass and small $q$ orientational dynamics, respectively. The topology of the phase diagram (Fig. 22) is related to the special form of the memory kernels (Eq. (50) and (51)), i.e. $m_{00}^{\alpha \alpha^{\prime}}$ does not only involve $S_{00}\left(q_{1}, t\right) S_{00}\left(q_{2}, t\right)$ but contains also a feedback from the dipolar motion, due to $S_{1 m_{1}}\left(q_{1}, t\right) S_{1 m_{2}}\left(q_{2}, t\right)$. In contrast $m_{1 m}^{\alpha \alpha^{\prime}}(q, t)$ only possesses a coupling between the center of mass and the dipolar correlators, i.e. the latter can only become nonergodic if the center of mass correlator becomes frozen. A schematic model which exactly describes this behavior is the Bosse-Krieger model [77]

$$
\begin{aligned}
\ddot{\phi}_{a}(t) & +\Omega_{a}^{2} \phi_{a}(t)+\nu_{a} \dot{\phi}_{a}(t)+ \\
& +\Omega_{a}^{2} \int_{0}^{t} d t^{\prime} m_{a}\left(t-t^{\prime}\right) \dot{\phi}_{a}\left(t^{\prime}\right)=0
\end{aligned}
$$

$a=0,1$ with the memory kernels

$$
\begin{aligned}
& m_{0}(t) \equiv F_{0}\left(\phi_{0}(t), \phi_{1}(t)\right)=\xi_{1} \phi_{0}^{2}(t)+\xi_{2} \phi_{1}^{2}(t)(60 \mathrm{a}) \\
& m_{1}(t) \equiv F_{1}\left(\phi_{0}(t), \phi_{1}(t)\right)=\xi_{3} \phi_{0}(t) \phi_{1}(t)
\end{aligned}
$$

and initial conditions $\phi_{a}(0)=1, \dot{\phi}_{a}(t)=0$. To mimic the $\phi$ - and $T$-dependence of the dipolar hard spheres we choose

$$
\xi_{1} \equiv \phi, \quad \xi_{2} \equiv x / T, \quad \xi_{3} \equiv 1 / T .
$$

This choice is motivated by the observation that the mode coupling between center of mass and dipolar dynamics vanishes for $T \rightarrow \infty$, leaving only the cage effect for simple hard spheres with "packing fraction" $\phi . x$ is considered as an additional parameter. From (59) and (60) we immediately get the equations

$$
\frac{f_{a}}{1-f_{a}}=F_{a}\left(f_{0}, f_{1}\right), \quad a=1,2
$$

for the corresponding non-ergodicity parameters

$$
f_{a}=\lim _{t \rightarrow \infty} \phi_{a}(t)
$$

(62) has been solved for $x=0.15$. From this solution we get the phase diagram for glass transitions presented in Figure 18. Comparison with Figure 2 reveals qualitative agreement between both phase diagrams. We emphasize that this result holds only in a certain range for $x$. The full $\xi_{1}-\xi_{2}-\xi_{3}$ or equivalently $\phi-T-x$ diagram was explored in detail in Ref. [78]. Since $f_{1}=0$ in the glass phase II (see Figure 18), Eqs. (62) for $a=0$ reduce to the $F_{2}$-model [1] for which the critical value for $\phi$ is $\phi_{c}^{F_{2}}=4$ and the critical non-ergodicity parameter is $f_{c}^{F_{2}}=\frac{1}{2}$ (see horizontal line in Figure 19).

Having found the same qualitative phase diagram as for dipolar hard spheres we can now investigate the dynamics for the Bosse-Krieger model. This has already been done in order to calculate the long time dynamics for the $\alpha$-relaxation [73] and the $\alpha$ - and $\beta$-relaxation [79]. An investigation which also includes the microscopic frequency regime can be found in Ref. 80]. Figure 4b of this Reference gives a hint for the existence of an extra peak between the $\alpha$ - and the hf-peak. But this phenomenon has not been discussed there.

In Figure 19 we present $\phi_{0}(t)$ for fixed frequency $\Omega_{0}=$ 1 and different values for $\Omega_{1}$. For $\Omega_{1}=\Omega_{0}$ we find the typical two step relaxation process from $\phi_{0}(0)=1$ to a plateau at $f_{c} \equiv f_{c}^{F_{2}}$ and a final decay to zero. With decreasing $\Omega_{1}$ we observe that a shoulder occurs which finally develops into a second plateau above $f_{c}^{F_{2}}$ This can easily be understood. In case that $\Omega_{1}$ becomes more and more soft, with respect to $\Omega_{0}$, the second term in $m_{0}(t)$ (cf. Eq. (60a) varies slower and slower, already on the microscopic time scale $t_{0}=\Omega_{0}^{-1}$. Therefore the relaxation kernel $m_{0}(t)$ has a rather slowly varying part. It is this part which generates the second plateau. Since the decay from a pronounced plateau produces a relaxation peak in the corresponding susceptibility spectrum we expect, besides the high frequency peak related to $\Omega_{0}$ and the $\alpha$-peak, one more peak. This peak stems from the decay from the upper plateau to that at $f_{c}^{F_{2}}$. Figures 20 and 21 present $\chi_{a}^{\prime \prime}(\omega)$ for $\Omega_{0}=1, \Omega_{1}=0.1$ and $\nu_{0}=\nu_{1}=0$ along path A' and B' (see Figure 18), respectively. Starting in the ergodic phase not too close to the transition line there is in $\chi_{0}^{\prime \prime}(\omega)$ the $\alpha$-peak (visible on the linear scale for $\epsilon<0$ and $n=1$, only) and the high frequency peak at $\omega \approx \Omega_{0}$, only. Approaching the critical line, we observe that the high frequency peak shifts to higher frequencies, due to "stiffening" (see Ref. [57]), and the $\alpha$-peak to lower frequencies. With this shift of the $\alpha$-peak, a third peak becomes more pronounced at a position which is about the same as the position of the main peak in $\chi_{1}^{\prime \prime}(\omega)$ and which scales with the bare frequency $\Omega_{1}$. This peak remains also in the glass phase. To investigate the nature of that additional peak again we have plotted $\phi_{a}^{\prime \prime}(\omega)$ in Figure 22 and 23 for path A' and B', respectively. The hf-peak occurs already in the "liquid" phase (at least for the lower "temperature") indicating a damped oscillatory dynamics. The spectral 
shape of the hf-peak strongly resembles the shape of the anomalous oscillation peak (AOP) found in Ref. [57] for a hard sphere system, a one-and a two component schematic model. Therefore our notation hf-peak should not be confused with the high-frequency sound peak in Ref. [57]. In contrast, the additional peak at $\omega \approx \Omega_{1}$ does not exist in the "liquid" but in the "glass" phase, only. Hence, the character of the extra peak changes from a relaxational to a damped oscillational type. Therefore the occurrence and the qualitative features of the additional peak are quite similar to the behavior found for the system of dipolar hard spheres. Since $\Omega_{1}$ mimics the orientational frequency $\Omega_{1 m}(q)$, we have further support of the orientational origin of the orientational peak found in $\chi_{00}^{\prime \prime}(q, \omega)$ and $\phi_{00}^{\prime \prime}(q, \omega)$ for dipolar hard spheres. In addition, the schematic model also demonstrates that an additional microscopic low frequency peak can only exist when the microscopic dynamics for $\phi_{1}(t)$ is "soft" enough with respect to that of $\phi_{0}(t)$.

For completeness we mention that for the lower "temperature" (path A') there exists in $\phi_{0}^{\prime \prime}(\omega)$ a further peak at $\omega \approx 0.4$. This peak becomes more prominent on a double-logarithmic representation (see inset of Fig.22) and its position also scales with $\Omega_{1}$, and not with $\Omega_{0}$. Hence, both peaks at $\omega \approx 0.1$ and $\omega \approx 0.4$ have their origin in the "orientational" motion of $\phi_{1}(t)$ and its coupling to $\phi_{0}(t)$ via mode coupling terms. Performing a "stiff-glass"-approximation [57] for the BK-model (which has not yet been done) should allow to elucidate the peak structures and the spectral shapes more in detail. Since this is not the purpose of the present paper this will be discussed elsewhere.

It is also instructive to neglect memory effects for $\phi_{1}(t)$, i.e. we put $m_{1}(t)$ equal to zero. Then, (59) for $a=1$ describes a damped harmonic oscillator with a feedback on $\phi_{0}(t)$ due to the second term in $m_{0}(t)$. For this "reduced" Bosse-Krieger model we present $\chi_{0}^{\prime \prime}(\omega)$ in Figure 24 for $\Omega_{0}=1, \Omega_{1}=0.1, \nu_{0}=0$ and $\nu_{1}=0.1$. Note that we introduced a nonzero damping $\nu_{1}$ in order to comply with the nonzero width of the main peak in $\chi_{1}^{\prime \prime}(\omega)$ for the original BK-model. As expected one observes an additional peak which originates from the damped harmonic motion of $\phi_{1}(t)$. Such an observation has already been made earlier for another type of schematic model [81,54]. Comparing $\chi_{0}^{\prime \prime}(\omega)$ from Figure 21 and $\phi_{0}^{\prime \prime}(\omega)$ from Figure 23 with $\chi_{0}^{\prime \prime}(\omega)$ and $\phi_{0}^{\prime \prime}(\omega)$ in Figure 24 shows that the memory effects for $\phi_{1}(t)$ do not influence $\chi_{0}^{\prime \prime}(\omega)$ and $\phi_{0}^{\prime \prime}(\omega)$ qualitatively.

\section{DISCUSSION AND CONCLUSIONS}

Recently we have extended the mode coupling theory (MCT) for simple or binary 1 - 4 to molecular liquids [6, 76, 68]. This has been done within a molecular representation, which separates translational from orientational degrees of freedom. This molecular theory has already been applied and tested for diatomic molecules $82,12,13$ and water molecules with SPC/E potential [7, 14]. These tests were restricted to the comparison of the nonergodicity parameters and critical amplitudes (for diatomic molecules, only) from molecular MCT with the corresponding quantities from a MD-simulation. A satisfactory agreement between MCT and MD-simulation has been found So far no dynamical results were determined from mode coupling theory for molecular liquids. The only solution of the time-dependent MCT-equations for a system with orientational degrees of freedom has been obtained recently for the orientational correlators of a single dumbell in an isotropic liquid 67,56. In the present paper we have solved the time-dependent molecular MCT equations for a system of dipolar hard spheres. This is one of the simplest systems involving translational and orientational degrees of freedom. In addition it has the advantage, that the static correlators which are the input quantities for the MCT-equations are known analytically within some approximations. These approximations make the tensorial correlators in the $q$-frame $\phi_{l m, l^{\prime} m^{\prime}}\left(\vec{q}=\vec{q}_{0}, t\right)=\phi_{l m}(q, t) \delta_{l l^{\prime}} \delta_{m m^{\prime}}$ diagonal in $l$ and $l^{\prime}$. In addition they lead to a restriction of $\phi_{l m}(q, t)$ to $l=0,1$ only. This means that our calculations yield information on center of mass and dipolar dynamics.

The major result we have found in the microscopic frequency regime is the existence of an orientational peak at $\omega_{o p}($ liquid $) \approx \hat{\omega}_{o p}$ (glass) about one decade below the high frequency peak. This orientational peak exists above and below the glass transition lines in the two dimensional phase diagram of a dimensionless temperature $T^{*}$ and packing fraction $\phi$ provided that the $\alpha$-peak position $\omega_{\alpha}$ is much smaller than $\omega_{o p}$. On the liquid side it exists in $\chi_{00}^{\prime \prime}(q, \omega)$, the compressibility spectrum but not in $\phi_{00}^{\prime \prime}(q, \omega)$ which proves its pure relaxational character. However, crossing the glass transition line this peak also appears in the correlation spectrum $\phi_{00}^{\prime \prime}(q, \omega)$ if $T^{*}$ and $\phi$ becomes, respectively, small and large enough. This means that the orientational peak exists on both sides of the glass transition lines and changes from a relaxational to a damped oscillational type of excitation under transformation of the liquid to the glass. The manner how the op develops in $\chi_{00}^{\prime \prime}(q, \omega)$ as a function of $\phi$ (see the double log representation in Fig. 6) resembles at least qualitatively, the behavior of the extra peak in the susceptibility spectra for e.g. Salol and Orthoterphenyl [83,20,84] at $\omega \approx 300 \mathrm{GHz}$ under variation of the temperature.

Since the position $\omega_{o p}$ is almost independent on $q$ it is related to a localized, non-propagating mode. In addition, the isotope-effect, i.e. $\omega_{o p} \propto I^{-1 / 2}$, clearly proves its orientational origin. Since the bare frequencies $\Omega_{l m}(q)$ for $l=1$ scale with $I^{-1 / 2}$ for $q=0$, only (cf. Eq. (54)), the orientational peak must be generated by orientational modes with $q \approx 0$. Why do so long-waved orientational modes play a crucial role? An answer follows from the static correlator $S_{11}(q)$. In contrast to $S_{00}(q)$ and $S_{10}(q)$ it exhibits a dominant peak with width $\Delta q$ at $q=0$ and decays rapidly to one for $q>\Delta q$ (see Fig. 11). With 
decreasing temperature its peak height increases and $\Delta q$ decreases. The increase of $S_{11}(q \approx 0)$ has two implications:

(i) since the glass transition in $\mathrm{MCT}$ is driven by the growing of the main peaks of the static correlators [1 it is the increase of $S_{11}(q \approx 0)$ and the coupling of the orientational mode $\rho_{11}(\vec{q}, t)$ to $\rho_{00}(\vec{q}, t)$, the translational one, which enhances the tendency for glass formation. This type of glass transition mechanism was already described within the framework of MCT for crystalline systems. For orientational glasses [85,86] and strictly periodic lattices 87.88 which undergo a second order equilibrium phase transition the increase of the critical fluctuations already lead to an ideal glass transition above the equilibrium transition temperature. The increase of the critical fluctuations is accompanied by the softening of the critical mode.

(ii) since $\Omega_{11}(q) \propto 1 / \sqrt{S_{11}(q)}$ (cf. Eq. (54)) the increase of $S_{11}(q \approx 0)$ implies a softening of the orientational mode for $q \approx 0$ (see also Fig. 3). It is this softening of the orientational mode with $q \approx 0$ and $l=1$, $m=1$ which results in a frequency scale separation between this orientational mode and the translational one $(l=0, m=0)$ with $q \approx q_{\max }$. Therefore two peaks, the orientational and the hf-peak, become visible. The connection of $\hat{\omega}_{o p}(q)$ with the $(1,1)$-mode is also supported by the fact that $\hat{\omega}_{o p}(q) \approx \hat{\Omega}_{11}^{ \pm}(q)$ (see Fig. 价).

The narrowing of the peak in $S_{11}(q)$ at $q=0$ also has an interesting implication. Since $1 / \Delta q$ is a measure of a correlation length, the narrowing implies a growth of an orientational order. Although $1 / \Delta q$ does not diverge, it may be significantly larger than a few Angström, i.e there may exist a medium range orientational order. For instance, for $\phi=0.381$ and $T^{*}=0.04$ the correlation length is about six diameters (cf. Figure 1). The fact that the increase of $S_{11}(q \approx 0)$ is accompanied by the narrowing of the peak in $S_{11}(q)$ at $q=0$ proves the existence of a correlation between the occurrence of the orientational peak and medium range orientational order. This is an interesting observation insofar that such a correlation has already been predicted 889 91, 39, 92, although this does not seem to be a universal feature [93]. We emphasize that we do not consider this type of behavior as an exceptional case which exists for dipolar hard spheres, only. For example for a system of hard ellipsoids which may exhibit medium ranged nematic order (see Ref. [94]) similar results are expected.

Besides the op, we have found in $\chi_{00}^{\prime \prime}(q, \omega)$ and $\phi_{00}^{\prime \prime}(q, \omega)$ above and below the glass transition a high fre-

\footnotetext{
${ }^{1}$ That the underlying mechanism leading to an ideal glass transition can be much more sophisticated, i.e. not only related to the increase of e.g. one peak in $S_{00}(q)$ has been recently demonstrated for a system of hard spheres with attractive interactions 99102
}

quency peak at $\omega_{h f}(q)$. It exists in the investigated range $0.51<q<40$ except in an interval centered around $q_{\max }$, the position of the main peak in $S_{00}(q)$. $\omega_{h f}(q)$ is shown in Figure 1 for $q \leq 2$. Despite an inaccuracy of about 10 (due to the $q$-discretization) its $q$-dependence is in phase with the $q$-variation of the renormalized frequency $\hat{\Omega}_{00}(q)$. These qualitative features are similar to the type of the high frequency peak found for a system of hard spheres [57]. Since the accuracy of $\omega_{h f}(q)$ becomes even worse for $q \rightarrow 0$ it is not possible to detect the expected linear $q$-dependence for $q \rightarrow 0$. Accordingly, we can not yet prove that the hf-peak for DHS corresponds to the high frequency sound, as found for the system of hard spheres [57]. In [57] it has also been shown that an additional peak, which was called "anomalous oscillation peak" (AOP), appears deep in the glass phase. Its origin lies in the harmonic motion of the particles in their cages [57]. Whether or not the hump between the op and the hf-peak we have observed in $\phi_{00}^{\prime \prime}(q, \omega)$ deep in the glass (see Fig. 10 and 15) corresponds to the AOP is not clear. Again due to the restriction to 30 values for $q$ and also a smaller number of discrete values for $t$ compared to Ref. 57] we can not give detailed quantitative information on, e.g. the $q$-dependence of the position and width of that hump. On the other hand the high frequency wing of the orientational peak may interfere with the AOP, thus complicating the analysis of the latter.

A well-pronounced two-peak structure in the microscopic frequency regime can also occur for $\chi_{10}^{\prime \prime}(q, \omega)$ (cf. Fig. 7) deep in the glass, whereas this does not happen for $\chi_{11}^{\prime \prime}(q . \omega)$ (cf. Fig. 1) which is directly related to the dielectric loss spectrum $\epsilon^{\prime \prime}(q, \omega)$.

Let us come back to the orientational peak. Figure $17 \mathrm{a}$ shows that its intensity varies linearly with temperature below about $T^{*}=0.13$ and for $\phi_{D}=0.525$, i.e. for $T^{*}$ smaller than $T_{c}^{*}\left(\phi_{D}=0.525\right) \simeq 0.13$. This fact, as well as the rather insensitivity of its position $\omega_{o p}$ on $q$ and its location about one decade below the high frequency peak is reminiscent to the boson peak. Additionally the increase of $\omega_{o p}$ with packing fraction is similar to the shift of the boson peak to higher frequency by increasing the pressure [95 97].

To conclude we can say that we have found in the microscopic frequency regime of the compressibility spectrum and the corresponding correlation spectrum an additional orientational low frequency peak. It originates from a localized and non-propagating orientational mode coupled to the longitudinal acoustic sound waves (translational modes). Its dynamical origin changes from a pure relaxational to a damped oscillational type when crossing the glass transition from the liquid side. Since the orientational frequency has a gap at $q=0$ the orientational excitation is an optical mode. These characteristic features coincide with several explanations for the existence of the boson peak [18, 35, 58, 62, 63] which may stress the role of orientational degrees of freedom. It is also interesting that the intensity of the op in a certain temperature regime (cf. Fig. 17a) changes linear with 
$T^{*}$ which is also true for the boson peak. In addition we have found an interesting correlation between the orientational peak phenomenon and a medium range orientational order, as already suggested earlier for the boson peak 89 91.39.92], although it has been shown that this does not seem to hold for all glass formers [93].

For dipolar hard spheres this order is of ferroelectric type and for hard ellipsoids related to a precursor of nematic order. For $\mathrm{SiO}_{2}$ it can occur due to strong orientational bond interactions. As demonstrated in the present paper this medium range orientational order may result in a softening of a localized, optic orientational mode and may finally generate an additional microscopic peak about one decade below the high frequency peak.

In general it is not so easy to separate from experimental spectra obtained from light or neutron scattering the contribution from the orientational motion. But this is not true for a MD-simulation. Due to the availability of all the microscopic information it would be desirable to explore in more detail by MD-simulations the role of the orientational degrees of freedom for the glass transition itself and also for the spectra in the microscopic regime, as it has already been done for the sound propagation in liquid water 98].

Finally we mention that the microscopic spectral features we have found for DHS can be qualitatively reproduced by use of a schematic model, the Bosse-Krieger model.

ACKNOWLEDGMENTS We are grateful to W. Götze for his careful reading of this manuscript and many valuable comments, to M. Sperl and Th. Voigtmann for their discussion with respect to the accuracy of our numerical results and to P. A. Madden for the discussion on orientational modes in molecular liquids. The financial support by Sonderforschungsbereich 262 is gratefully acknowledged, as well.

[1] W. Götze in, Liquids, Freezing and the Glass transition, (edited by J. P. Hansen, D. Levesque and J. Zinn-Justin, North-Holland, Amsterdam 1991).

[2] W. Götze and L. Sjögren, Rep. Prog. Phys. 55, 241 (1992).

[3] R. Schilling, Disorder Effects on Relaxation Processes, (edited by R. Richter and A. Blumen, Springer, Berlin 1994).

[4] H. Z. Cummins, J. Phys. Cond. Mat. 11, A95 (1999).

[5] T. Franosch, M. Fuchs, W. Götze M. R. Mayr and A. P. Singh, Phys. Rev. E 56, 5659 (1997).

[6] R. Schilling and T. Scheidsteger, Phys. Rev. E 56, 2932 (1997).

[7] L. Fabbian, A. Latz, R. Schilling and F. Sciortino, P. Tartaglia, C. Theis, Phys. Rev. E 60, 5768 (1999).
[8] Transp. Theory Stat. Phys. 24, 6-8, special issue on relaxation kinetics in supercooled liquids-mode-coupling theory and its experimental tests, edited by S. Yip (1995).

[9] W. Kob, J. Phys. Cond. Mat 11, R85 (1999).

[10] W. Götze, J. Phys.: Condens. Matter 11, A1 (1999).

[11] N. Tao, G. Li, X. Chen, W. Du and H. Cummins, Phys. Rev. A 44, 6665 (1991).

[12] R. Schilling, J. Phys.: Condens. Matter 12, 6311 (2000).

[13] A. Winkler, A. Latz, R. Schilling and C. Theis, Phys. Rev. E 62, 8004 (2000).

[14] C. Theis, F. Sciortino, A. Latz, R. Schilling and P. Tartaglia, Phys. Rev. E 62, 1856 (2000).

[15] C. Theis and R. Schilling, J. Non-Cryst. Solids 235-237, 106 (1998).

[16] R. O. Pohl, Amorphous Solids-Low Temperature Properties, Vol. 24 of "Topics in Current Physics" (edited by W. A. Phillips, Springer, Berlin 1981).

[17] V. K. Malinovski and A. P. Sokolov, Solid State Commun. 57, 757 (1986).

[18] U. Buchenau, N. Nücker and A. J. Dianoux, Phys. Rev. Lett. 53, 2316 (1984).

[19] U. Buchenau, M. Prager, N. Nücker, A. J. Dianoux, N. Ahmad and W. A. Phillips, Phys. Rev. B 34, 5665 (1986).

[20] H. Z. Cummins, G. Li, W. Du, Y. H. Hwang and G. Q. Shen, Prog. Theor. Phys. Suppl. No. 26, 21 (1997).

[21] M. C. C. Ribeiro, M. Wilson and P. A. Madden, J. Chem. Phys. 109, 9859; 110, 4803, (1998; 1999).

[22] P. Benassi, M. Krisch, C. Masciovecchio, V. Mazzacurati, G. Monaco, G. Ruocco, F. Sette and R. Verbeni, Phys. Rev. Lett. 77, 3835 (1996).

[23] M. Foret, E. Courtens, R. Vacher and J. -B. Suck, Phys. Rev. Lett. 77, 3831 (1996).

[24] M. Foret, E. Courtens, R. Vacher and J. -B. Suck, Phys. Rev. Lett. 78, 4669 (1997).

[25] P. Benassi, M. Krisch, C. Masciovecchio, V. Mazzacurati, G. Monaco, G. Ruocco, F. Sette and R. Verbeni, Phys. Rev. Lett. 78, 4770 (1997).

[26] A. Matic, L. Björjesson, G. Ruocco, C. Masciovecchio, A. Mermet, F. Sette and R. Verbeni, Euro. Phys. Lett. 54, 77 (2001).

[27] C. Masciovecchio, A. Mermet, G. Ruocco and F. Sette, Phys. Rev. Lett. 85, 1266 (2000).

[28] O. Pilla, A. Cunsolo, A. Fontana, C. Masciovecchio, G. Monaco, M. Montagna, G. Ruocco, T. Scopigno and F. Sette, Phys. Rev. Lett. 85, 2136 (2000).

[29] J. Horbach, W. Kob and K. Binder, edited by M. R. Johnson et al. (AIP conference proceedings 479, $136(1999))$.

[30] J. Horbach, W. Kob and K. Binder, Eur. Phys. J. B 19 531 (2001).

[31] C. Masciovecchio, G. Ruocco, F. Sette, M. Krisch, R. Verbeni, U. Bergmann and M. Soltwisch, Phys. Rev. Lett. 76, 3356 (1996).

[32] M. Sampoli, G. Ruocco and F. Sette, Phys. Rev. Lett. 79, 1678 (1997).

[33] H. R. Schober and C. Oligschleger, Phys. Rev. B 53 11469, (1996). 
[34] S. N. Taraskin and S. R. Elliott, Europhys. Lett. 39, 37 (1997).

[35] S. N. Taraskin and S. R. Elliott, Phys. Rev. B 56, 8605 (1997).

[36] S. N. Taraskin and S. R. Elliott, Phys. Rev. B 59, 8572 (1999).

[37] S. N. Taraskin and S. R. Elliott, J. Phys.: Condens. Matter 11, A219 (1999).

[38] V. Mazzacurati, G. Ruocco and M. Sampoli, Europhys. Lett. 34, 681 (1996).

[39] M. Foley, M. Wilson and P. A. Madden, Phil. Mag. B 71, 557 (1995).

[40] M. C. C. Ribeiro, M. Wilson and P. A. Madden, J. Chem. Phys. 108, 9027 (1999).

[41] D. Engberg, A. Wischnewski, U. Buchenau, L. Börjeson, A. J. Dianoux, A. P. Sokolov and L. M. Torell, Phys. Rev. B 58, 9087 (1998).

[42] A. P. Sokolov, J. Phys.: Condens. Matter 11, A213 (1999).

[43] W. Schirmacher and M. Wagner in, Dynamics of Disordered Materials, edited by (D. Richter, A. J. Dianoux, W. Petry and J. Teixeira pp. 231-234, Springer, Berlin 1989).

[44] W. Schirmacher, G. Diezemann and C. Ganter, Phys. Rev. Lett. 81, 136 (1998).

[45] V. Martín-Mayor, G. Parisi and P. Verrocchio, Phys. Rev. E 62, 2373 (2000).

[46] M. Mézard, G. Parisi and A. Zee, cond-mat 9906135 (1999).

[47] V. G. Karpov, M. I. Klinger and F. N. Ignat'ev, Sov. Phys. JETP 57, 439 (1983).

[48] M. I. Klinger, Phys. Rep. 165, 275 (1988).

[49] Yu. M. Galperin, V. L. Gurevich and D. A. Parshin, Phys. Rev. B32, 6873 (1992).

[50] U. Buchenau, Yu. M. Galperin, V. L. Gurevich, D. A. Parshin, M. A. Ramos and H. R. Schober, Phys. Rev. B 46, 2798 (19).

[51] C. Alba-Simionesco and M. Krauzman, J. Chem. Phys. 102, 6574 (1995).

[52] W. Götze and L. Sjögren, Chem. Phys. 212, 47 (1996).

[53] T. Franosch, W. Götze M. R. Mayr and A. P. Singh, Phys. Rev. E 55, 3183 (1997).

[54] S. P. Das, Phys. Rev. E 59, 3870 (1999).

[55] B. Ruffle, C. Ecolivet and B. Toudic, Europhys. Lett. 45(5), 591 (1999).

[56] W. Götze, A. P. Singh and T. Voigtmann, Phys. Rev. E 61, 6934 (2000).

[57] W. Götze and M. Mayr, Phys. Rev. E 61, 587 (2000).

[58] B. Guillot and Y. Guissani, Phys. Rev. Lett. 78, 2401 (1997).

[59] A. Wischnewski, U. Buchenau, A. J. Dianoux, W. A. Kamitakahara and J. L. Zarestky, Phys. Rev. B 57, 2663 (1998).

[60] U. Schneider, P. Lunkenheimer, R. Brand und A. Loidl, Phys. Rev. E 59, 6924 (1999).

[61] P. Lunkenheimer, R. Brand, U. Schneider und A. Loidl, Contemporary Physics 41, 15 (2000).

[62] M. A. Ramos, S. Viera, E. J. Bermejo, J. Dawidowski, H. E. Fischer, H. Schober, M. A. Gonzalez, C. K. Loong and D. L. Price, Phys. Rev. Lett. 78, 82 (1997).
[63] H. E. Fischer, E. J. Bermejo, G. J. Cuello, M. T. Fernandez-Diaz, J. Davidowski, M. A. Gonzalez, H. Schober and H. Jimenez-Ruiz, Phys. Rev. Lett. 82, 1193 (1999).

[64] J. P. Hansen and I. R. McDonald, Theory of Simple Liquids (Academic Press, London, 2nd Edition, 1986).

[65] C. Theis and R. Schilling, Phys. Rev. E 60, 740 (1999).

[66] S.-H. Chong and F. Hirata, Phys. Rev. E 58(5), 6188 (1998).

[67] S.-H. Chong, W. Götze and A. P. Singh, Phys. Rev. E 63, 011206 (2000).

[68] A. Latz and M. Letz, European Journal of Physics 19, 3239 (2001)

[69] M. A. Ricci, D. Rocca, G. Ruocco and R. Vallauri, Phys. Rev. A 40, 7226 (1989).

[70] M.S. Wertheim, J. Chem. Phys. 55, 4291 (1971).

[71] C. G. Gray and K. E. Gubbins, Theory of Molecular Liquids (Clarendon, Oxford, 1984).

[72] A. Latz, PhD - Thesis Technische Universität München (1990).

[73] M. Fuchs, W. Götze, I. Hofacker and A. Latz, J. Phys. Condens. Matter 3, 5047 (1991).

[74] W. Götze and T. Voigtmann, Phys. Rev. E 61, 4133 (2000).

[75] T. Scheidsteger and R. Schilling, Phil. Mag. B 77, 305 (1998).

[76] T. Franosch, M. Fuchs, W. Götze M. R. Mayr and A. P. Singh, Phys. Rev. E 55, 7153 (1997).

[77] U. Krieger and J. Bosse, J. Phys. C 19, L609 (1986).

[78] W. Götze and R. Haussmann, Z. Phys. B 72, 403 (1988).

[79] Y. Kaneko and J. Bosse, J. Phys. Condens. Matter 8, 9581 (1996).

[80] J. Bosse, U. Krieger and J. S. Thakur, Dynamics of Disordered Materials, edited by (D. Richter, A. J. Dianoux, W. Petry and J. Teixeira pp. 231-234, Springer, Berlin 1989).

[81] T. Franosch, W. Götze M. R. Mayr and A. P. Singh, J. Non-Cryst. Solids 235-237, 71 (1998).

[82] C. Theis and R. Schilling, J. Non-Cryst. Solids 235 237, 106 (1998).

[83] H. Z. Cummins, W. M. Du, M. Fuchs, W. Götze, S. Hildebrand, A. Latz, G. Li and N. J. Tao, Phys. Rev. E 47(6), 4223 (1993).

[84] H. Z. Cummins, Y. H. Hwang, Gen Li, W. M. Du, W. Losert and G. Q. Shen, J. Non-Cryst. Solids 235237, 254 (1998).

[85] K. H. Michel, Z. Phys. B 71, 369 (1988).

[86] C. Bostoen and K. H. Michel, Phys. Rev. B 43, 4415 (1991).

[87] V. L. Aksenov, M. Bobeth, N. M. Plakida and J. Schreiber, J. Phys. C 20, 375 (1987).

[88] R. Schilling, Z. Phys. B 103, 463 (1997).

[89] S. R. Elliott, Phys. Rev. Lett. 67, 711 (1991).

[90] A. P. Sokolov, A. Kisliuk, M. Soltwisch and D. Quitmann, Phys. Rev. Lett. 69, 1540 (1992).

[91] A. P. Sokolov, A. Kisliuk, M. Soltwisch and D. Quitmann, Physica A 201, 295 (1993).

[92] R. Fayos, F. J. Bermejo, J. Dawidowski, H. E. Fischer and M. A. Gonzales, Phys. Rev. Lett. 77, 3823 (1996).

[93] L. Börjesson, A. K. Hassan, J. Swenson and L. M. Torell, 
Phys. Rev. Lett. 70, 1275 (1992).

[94] M. Letz, R. Schilling and A. Latz, Phys. Rev. E 625173 (2000).

[95] S. Sugai and A. Onodera, Phys. Rev. Lett. 77, 4210 (1996).

[96] Y. Inamura, M. Arai, O. Yamamuro, A. Inaba, N. Kitamura, T. Otomo, T. Matsua, S. M. Bennington and A. C. Hannon, Physica B 263, 299 (1999).

[97] P. Jund and R. Julien, cond-mat/0005169 (2000).

[98] F. Sciortino and S. Sastry, J. Chem. Phys. 100, 3881 (1994).

[99] L. Fabbian, W. Götze, F. Sciortino, P. Tartaglia and F. Thiery, Phys. Rev. E 59(2), R1347 (1999).

[100] E. Zaccarelli, G. Foffi, K. A. Dawson, F. Sciortino and P. Tartaglia, Phys. Rev. E 63 031501-1 (2001).

[101] K. Dawson, G. Floffi, M. Fuchs, W. Götze, F. Sciortino, M. Sperl, P. Tartaglia, T. Voigtmann and E. Zaccarelli, Phys. Rev. E 63, 0111401 (2001).

[102] J. Bergenholtz and M. Fuchs, Phys. Rev. E 59, 5706 (1999).

\section{APPENDIX A: THE NUMERICALLY SOLVED MCT EQUATIONS}

Using Eq. (4) we get the first equation of motion for the generalized density-density correlation functions (in the $q$-frame).

$$
\dot{S}_{l l^{\prime} m}(q, t)=-i \sum_{\alpha=R, T} q_{l}^{\alpha}(q) \phi_{l l^{\prime} m}^{j^{\alpha} \rho}(q, t)
$$

with

$$
\phi_{l l^{\prime} m}^{j^{\alpha} \rho}(q, t):=\frac{1}{N}\left\langle j_{l m}^{\alpha *}(q, t) \rho_{l^{\prime} m^{\prime}}(q, 0)\right\rangle \delta_{m m^{\prime}}
$$

the density - current-density correlation function. Projecting on the tensorial densities (Eq. (1) and the longitudinal translational and rotational current densities (Eq. (5)) at once leads to

$$
\begin{aligned}
& \dot{\phi}_{l l^{\prime} m}^{j^{\alpha} \rho}(q, t)= \\
& -i \sum_{l_{2}=m}^{l_{c o}} q_{l}^{\alpha}(q) \frac{k_{B} T}{I_{\alpha}}\left(\mathbf{S}^{-1}(q)\right)_{l l_{2} m} S_{l_{2} l^{\prime} m}(q, t) \\
& -\sum_{l_{2}=m}^{l_{c o}} \sum_{\alpha^{\prime}=R, T} \frac{k_{B} T}{I_{\alpha}} \int_{0}^{t} d t^{\prime} M_{l l_{2} m}^{\alpha \alpha^{\prime}}\left(q, t-t^{\prime}\right) \phi_{l_{2} l^{\prime} m}^{j^{\alpha^{\prime}} \rho}\left(q, t^{\prime}\right)
\end{aligned}
$$

with the cut-off value $l_{c o}$ for $l$ (for DHS it is $l_{c o}=1$, cf. Eqs. (48), (49)) and $I_{\alpha}$ defined in (16). To make use of the established numerical method to solve such equations 73] it is necessary to introduce auxiliary functions $\Phi$ :

$$
\dot{\Phi}_{l l^{\prime} m}^{j^{\alpha} \rho}(q, t):=q_{l}^{\alpha}(q) \phi_{l l^{\prime} m}^{j^{\alpha} \rho}(q, t)
$$

As a consequence Eq. (A1) reduces to

$$
S_{l l^{\prime} m}(q, t)=-i \sum_{\alpha=R, T} \Phi_{l l^{\prime} m}^{j^{\alpha} \rho}(q, t)
$$

where $\Phi_{l l^{\prime} m}^{j^{\alpha} \rho}(q, t)$ is determined only up to an integration constant which does not influence the result for $S_{l l^{\prime} m}(q, t)$. Making use of (A4) and taking the time derivative of Eq. (A3) leads to a set of equations of similar structure as the MCT equations for simple liquids [1, 2, 4, 3]. The molecular MCT - equations do not only couple all (discretized) wave vectors, as in simple liquids, but also the indices $\alpha=T, R$ for translation and rotation and the spherical indices $l \leq l_{c o}$ and $-l \leq m \leq l$. The functions $\Phi$ are auxiliary functions because their introduction is just for numerical purpose. The integral in Eq. (A3) transforms to

$$
\int_{0}^{t} d t^{\prime} M_{l l_{2} m}^{\alpha \alpha^{\prime}}\left(q, t-t^{\prime}\right) \dot{\Phi}_{l_{2} l^{\prime} m}^{j^{\alpha^{\prime}} \rho}\left(q, t^{\prime}\right) .
$$

Now a time derivative is multiplied with the integration measure $d t$. As a consequence the time step $\Delta t$ cancels at a discretized form of (A6 ). Using the decimation technique [73 $\Delta t$ becomes very large. In this case the calculation of the integrals would become unstable if $\Delta t$ would not cancel.

Since the auxiliary functions only occur as time derivatives in the original equations the result is independent on the initial values of $\Phi_{l l^{\prime} m}^{j^{\alpha} \rho}(q, t=0)$. When solving the molecular MCT equations we have chosen:

$$
\begin{aligned}
\Phi_{l l^{\prime} m}^{j^{T} \rho}(q, t=0) & =S_{l l^{\prime} m}(q), \\
\Phi_{l l^{\prime} m}^{j^{R} \rho}(q, t=0) & =0 \\
\text { for } l=0, \forall l^{\prime} \text { and } & \\
\Phi_{l l^{\prime} m}^{j^{T} \rho}(q, t=0) & =\frac{1}{2} S_{l l^{\prime} m}(q), \\
\Phi_{l l^{\prime} m}^{j^{R} \rho}(q, t=0) & =\frac{1}{2} S_{l l^{\prime} m}(q)
\end{aligned}
$$

for $l>0$ as initial values. 


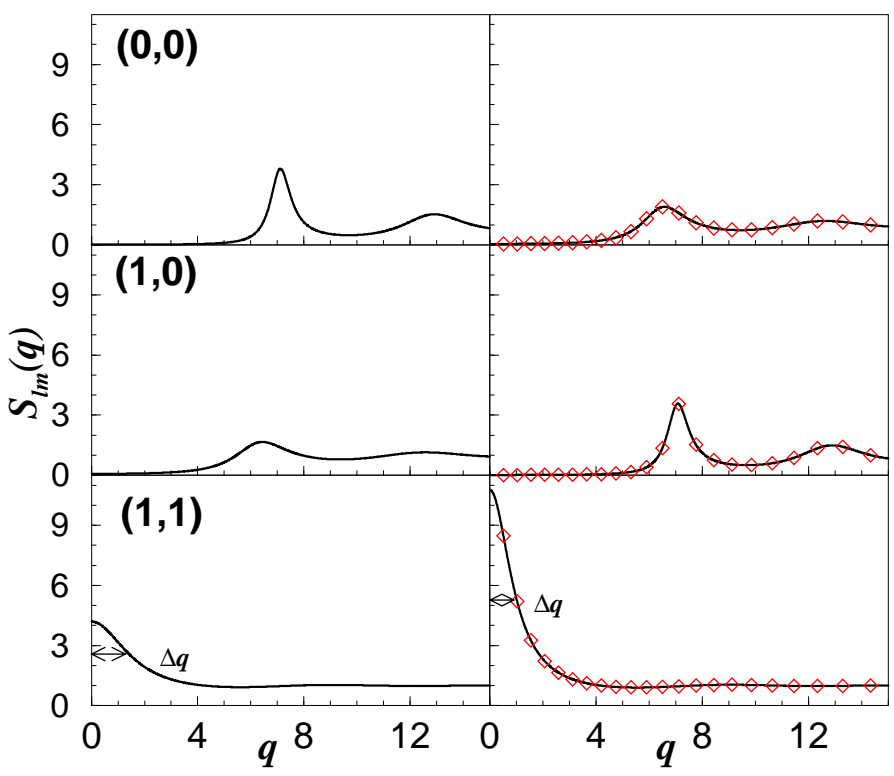

FIG. 1. The $q$ dependence of the static structure factors $S_{l m}(q)$ for $(l m)=(0,0),(1,0),(1,1)$ and two different pairs of $\left(\phi, T^{*}\right)$ indicated by the full circles in Figure 2: $(0.53,0.3)$ (left), $(0.381,0.04)$ (right). $\Delta q$ is the peak width of $S_{11}(q)$ at half maximum. The symbols indicate the discretized $q$-values (see text)

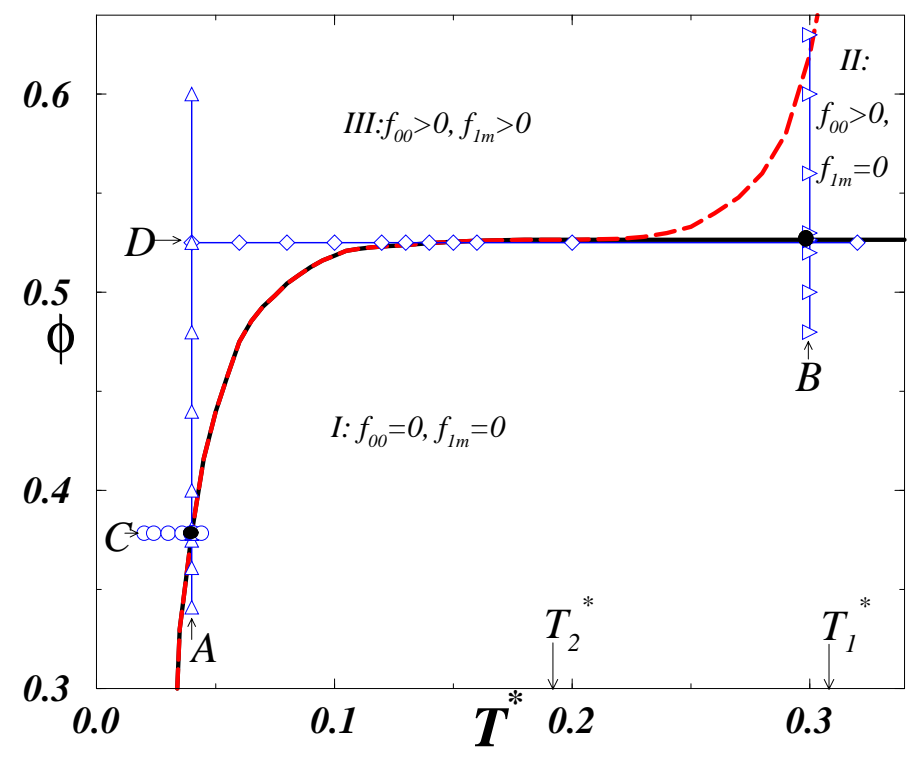

FIG. 2. Glass transition phase diagram for dipolar hard spheres: The solid and the dashed line are critical lines at which a discontinuous (type B) and a continuous (type A) glass transition takes place between the phases I,II and III. $\mathrm{A}, \mathrm{B}, \mathrm{C}$ and D denote the various paths on which we have investigated the control parameter dependence of the dynamics. The full circles indicate the two points at which the static correlators in Figure 1 and the microscopic frequencies in Figure 3 were calculated. For $T_{1}^{*}$ and $T_{2}^{*}$ see text

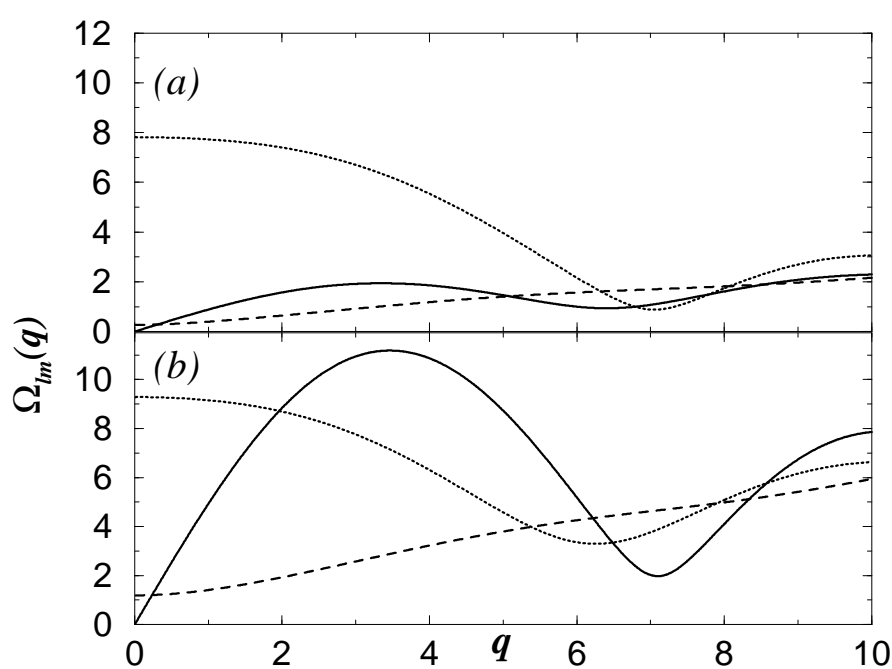

FIG. 3. $q$-dependence of the microscopic frequencies $\Omega_{l m}(q)$ for $(l, m)=(0,0)$ (solid), $(1,0)$ (dotted) and $(1,1)$ (dashed) (a) $\phi=0.381, T^{*}=0.04$ and (b) $\phi=0.53, T^{*}=0.3$, indicated in Figure 2 by the full circles

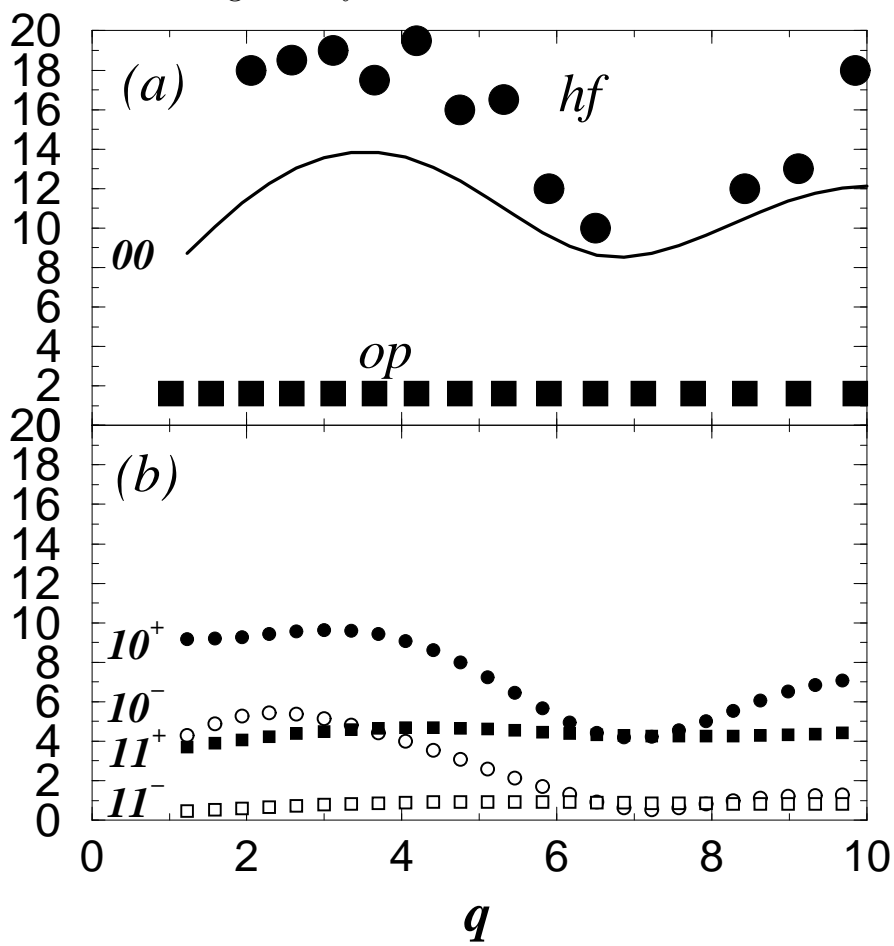

FIG. 4. $q$-dependence of the renormalized microscopic frequencies for $\phi=0.53, T^{*}=0.04$ : (a) $\hat{\Omega}_{00}(q)$ is shown as solid line; the position $\hat{\omega}_{h f}$ and $\hat{\omega}_{o p}$ obtained from $\phi_{00}^{\prime \prime}(q, \omega)$ are presented by the full circles and spheres, respectively (b) $\hat{\Omega}_{10}^{ \pm}(q)$ and $\hat{\Omega}_{11}^{ \pm}(q)$ are shown as circles and spheres, respectively. 


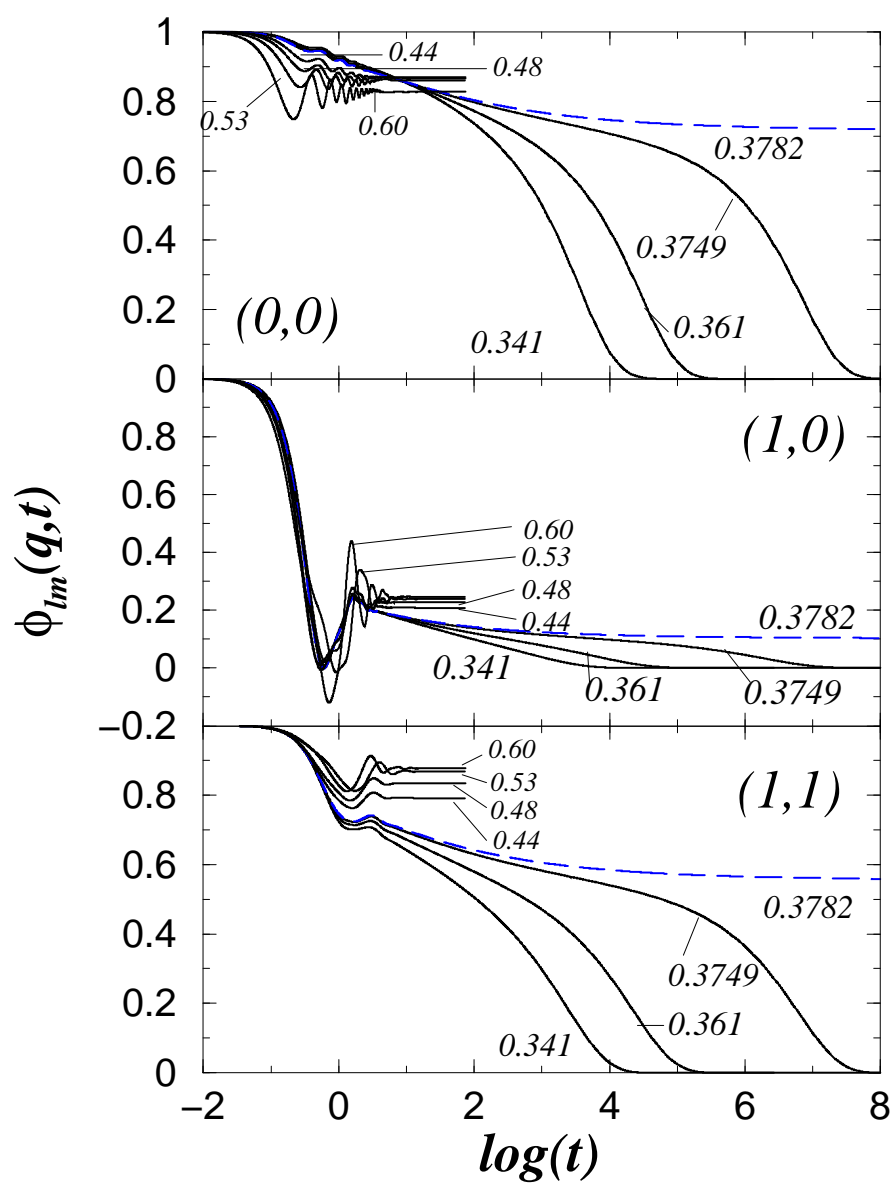

FIG. 5. lin-log plot of $\phi_{l m}(q, t)$ at $q \simeq 4.7, T_{A}^{*}=0.04$ and $\phi$ along path $\mathrm{A}$ in Figure 2. The dashed line corresponds to the results at $\phi \simeq \phi_{c} \simeq 0.3782$

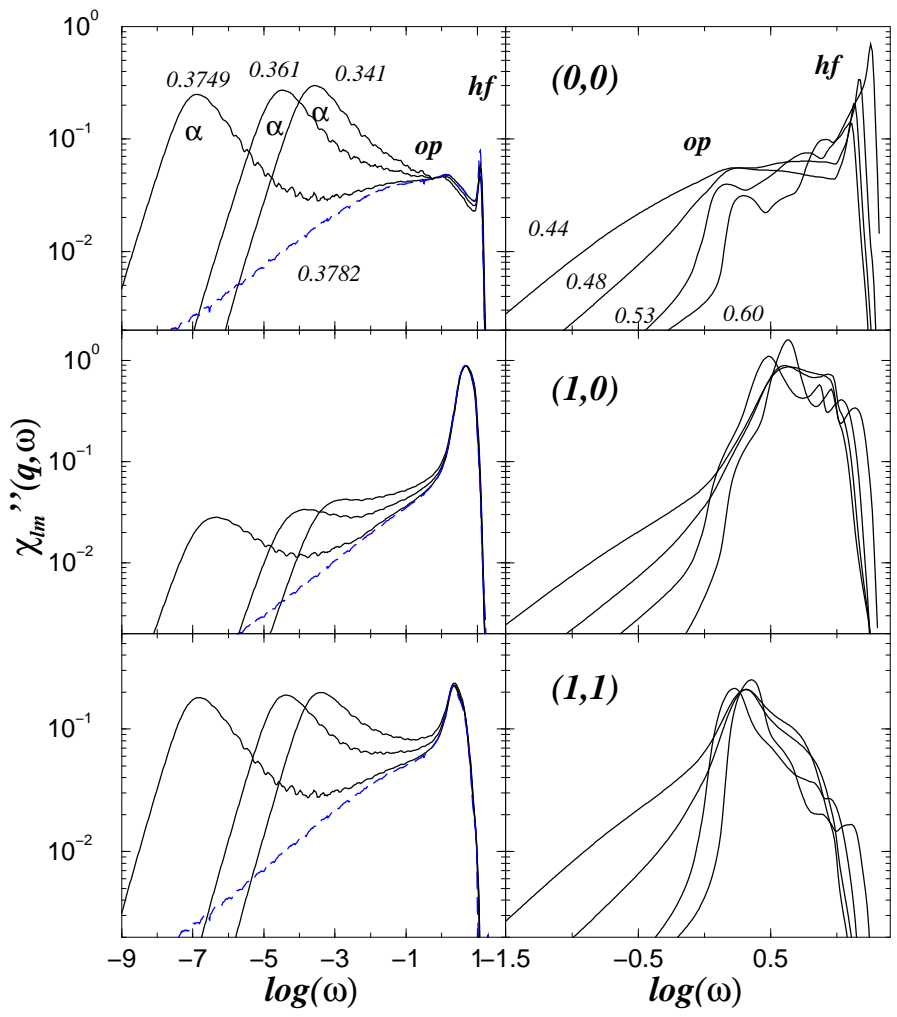

FIG. 6. log-log plot of $\chi_{l m}^{\prime \prime}(q, \omega)$ at $q \simeq 4.7, T_{A}^{*}=0.04$ and $\phi$ along path A in Figure 22 liquid (left column) and glass (right column). The dashed line corresponds to the results at $\phi \simeq \phi_{c} \simeq 0.3782$. $\phi$-values for $\chi_{10}^{\prime \prime}(q, \omega)$ and $\chi_{11}^{\prime \prime}(q, \omega)$ are the same as for $\chi_{00}^{\prime \prime}(q, \omega)$ 


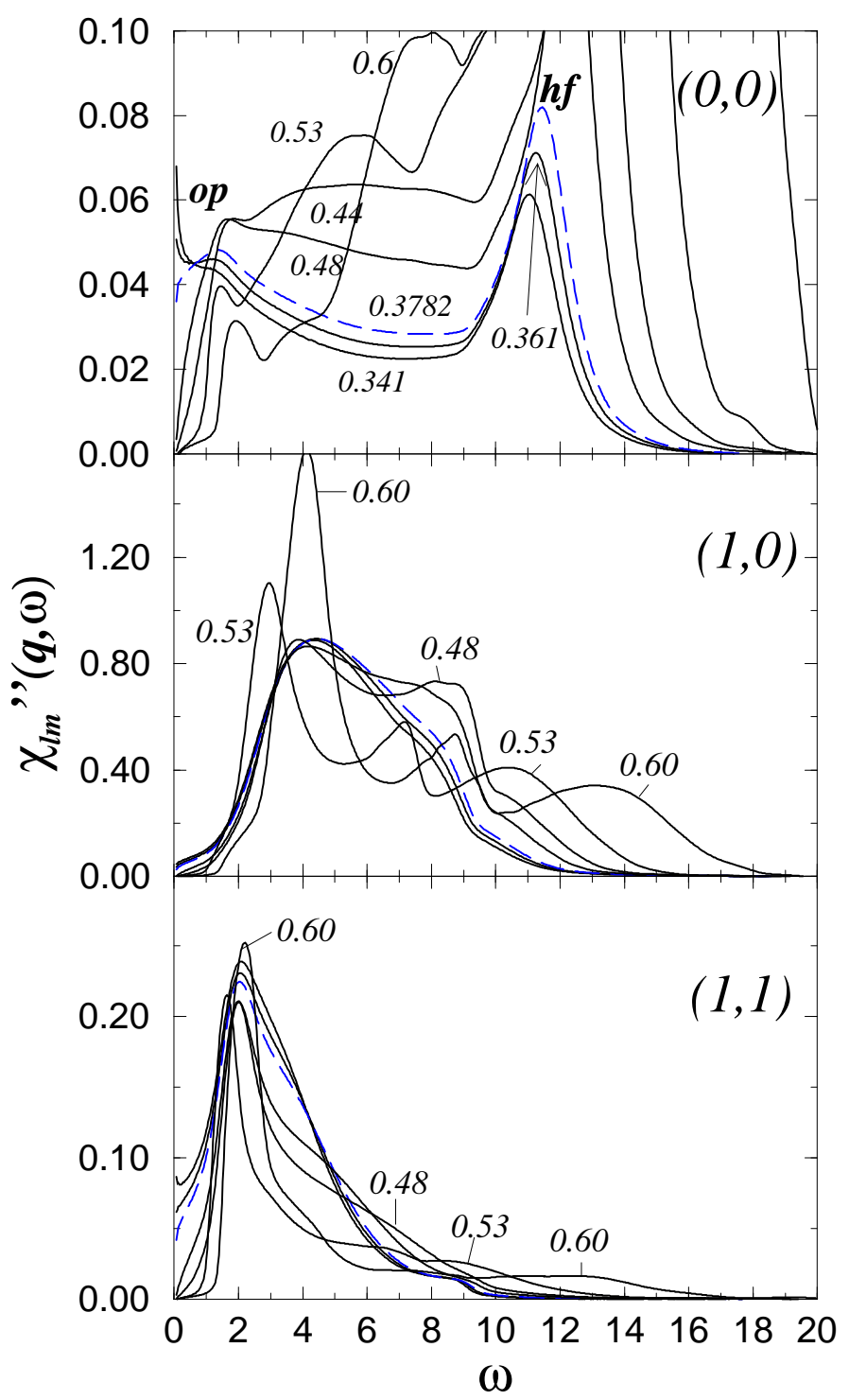

FIG. 7. $\chi_{l m}^{\prime \prime}(q, \omega)$ on linear scales at $q \simeq 4.7, T_{A}^{*}=0.04$ and $\phi$ along path $\mathrm{A}$ in Figure 2. The dashed line corresponds to the results at $\phi \simeq \phi_{c} \simeq 0.3782$

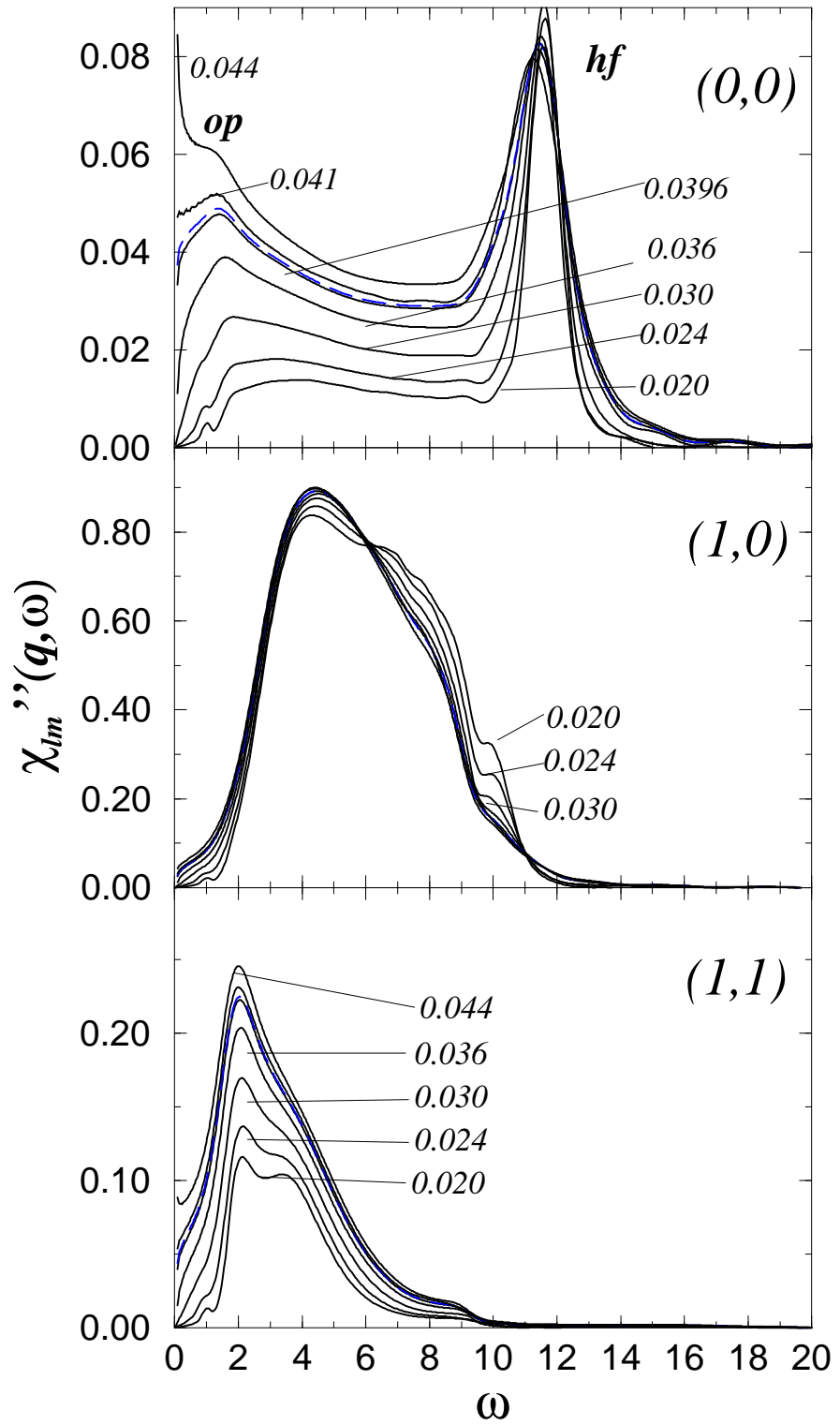

FIG. 8. $\quad \chi_{l m}^{\prime \prime}(q, \omega)$ on linear scales at $q \simeq 4.7, \phi_{C}=0.3786$ and $T^{*}$ along path $\mathrm{C}$. The dashed line corresponds to the result at $T^{*} \simeq T_{c}^{*} \simeq 0.04$ 


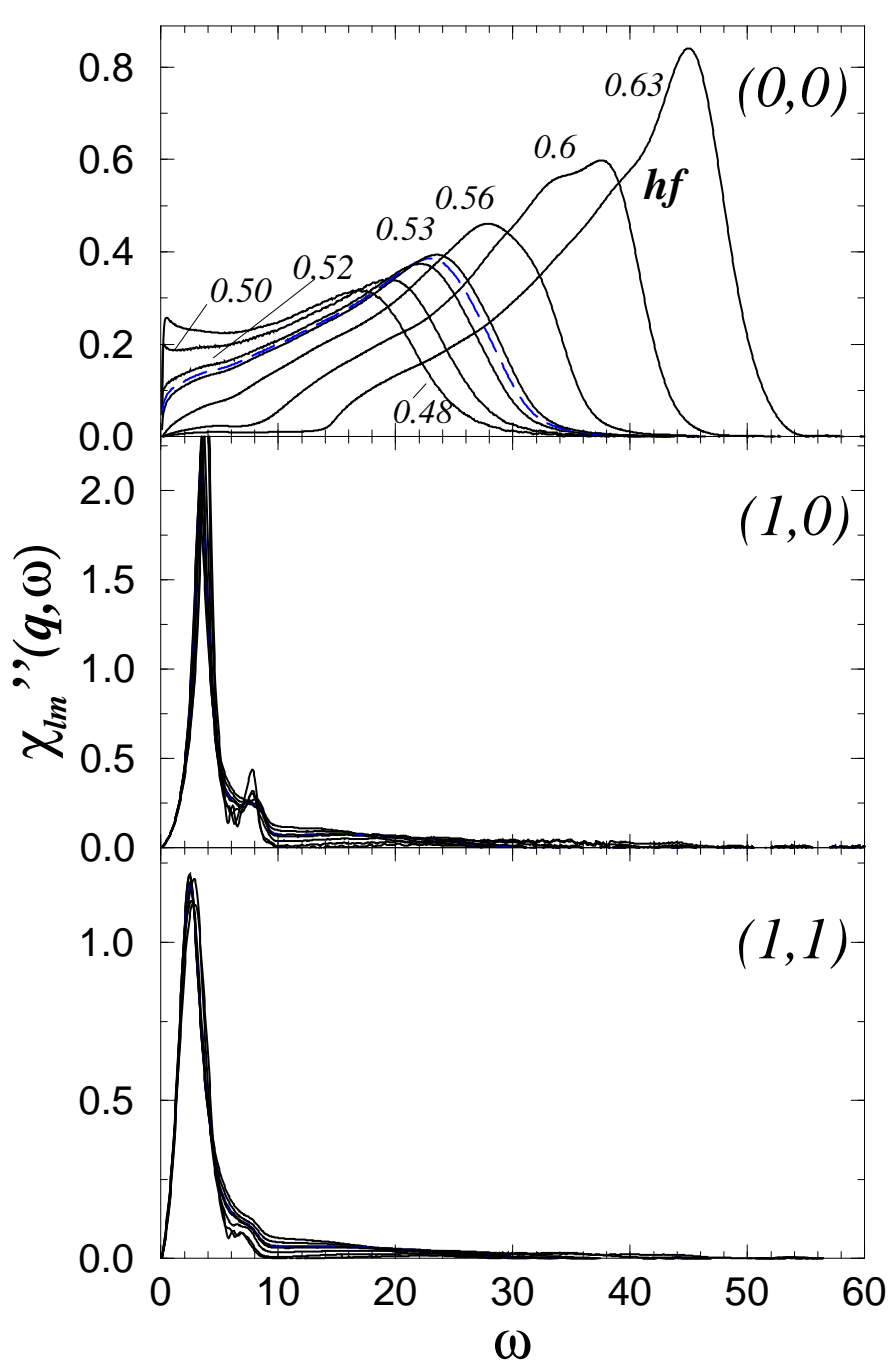

FIG. 9. $\quad \chi_{l m}^{\prime \prime}(q, \omega)$ on linear scales at $q \simeq 4.7, T_{B}^{*}=0.3$ and $\phi$ along path $\mathrm{B}$. The dashed line corresponds to the result at $\phi \simeq \phi_{c} \simeq 0.5265$. Because $\chi_{1 m}^{\prime \prime}(q, \omega)$ does not vary much with $\phi$ we have not labelled the various curves with $\phi$

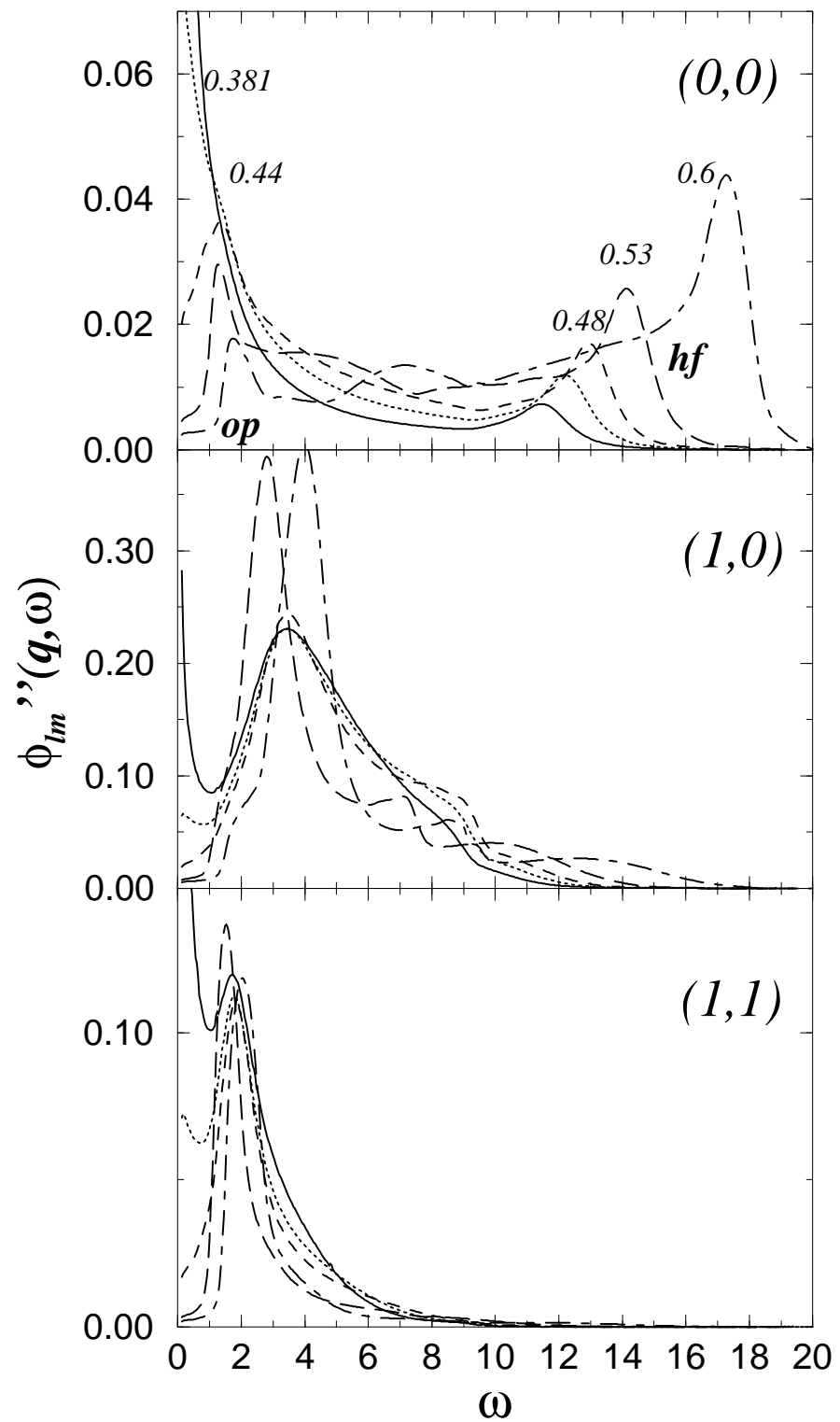

FIG. 10. $\phi_{l m}^{\prime \prime}(q \simeq 4.7, \omega)$ on linear scales in the glass phase along path A. I.e. $T^{*}=0.04$ and $\phi=0.381$ (solid), $\phi=0.44$ (dotted), $\phi=0.48$ (dashed), $\phi=0.53$ (long-dashed) and $\phi=0.6$ (dot-dashed) 


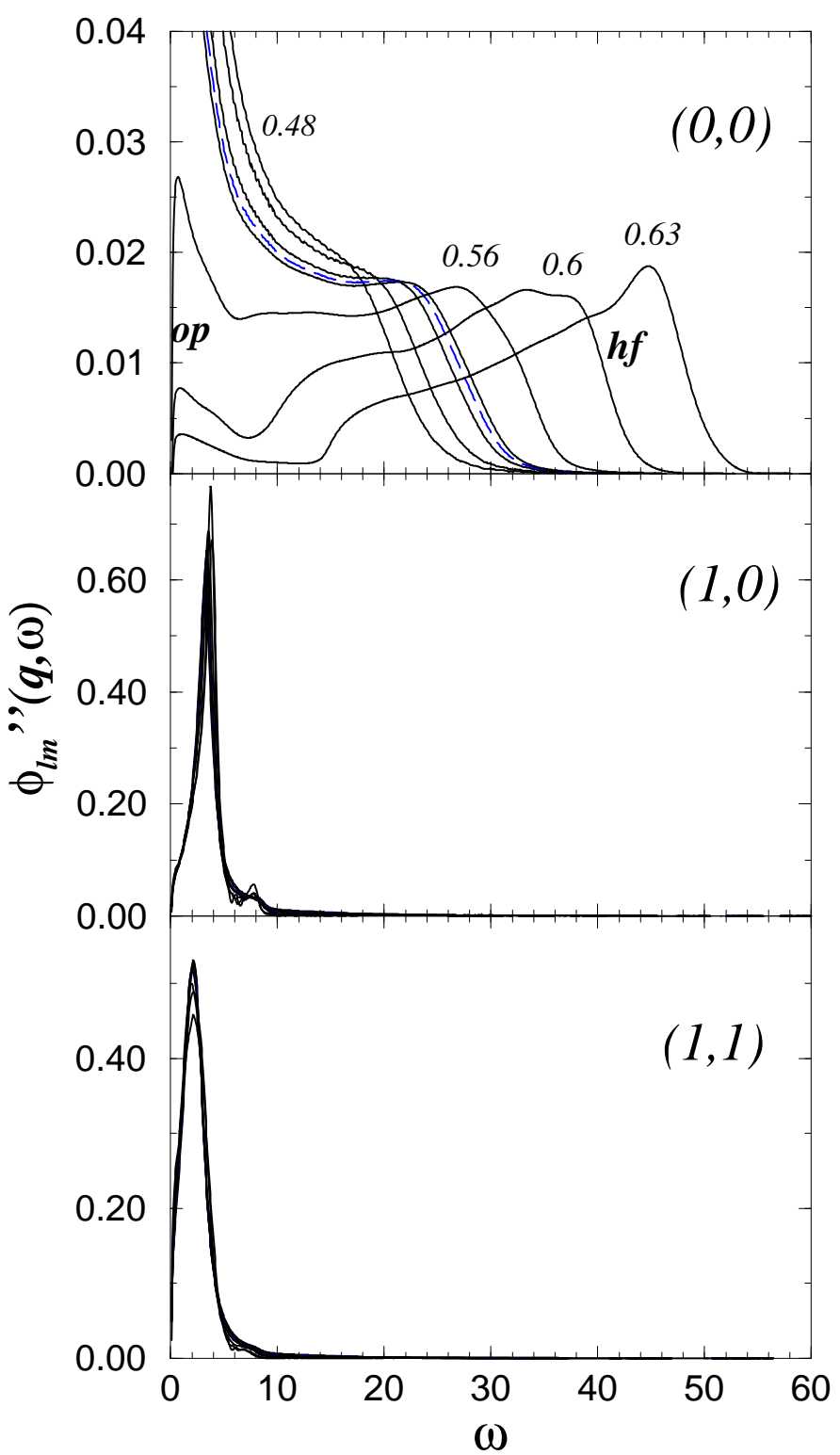

FIG. 11. $\phi_{l m}^{\prime \prime}(q \simeq 4.7, \omega)$ on linear scales for $T^{*}=0.30$ and $\phi$ along path B. The dashed line corresponds to the result at $\phi \simeq \phi_{c} \simeq 0.5265$. Because $\phi_{1 m}^{\prime \prime}(q, \omega)$ does not vary much with $\phi$ we have not labelled the various curves with $\phi$

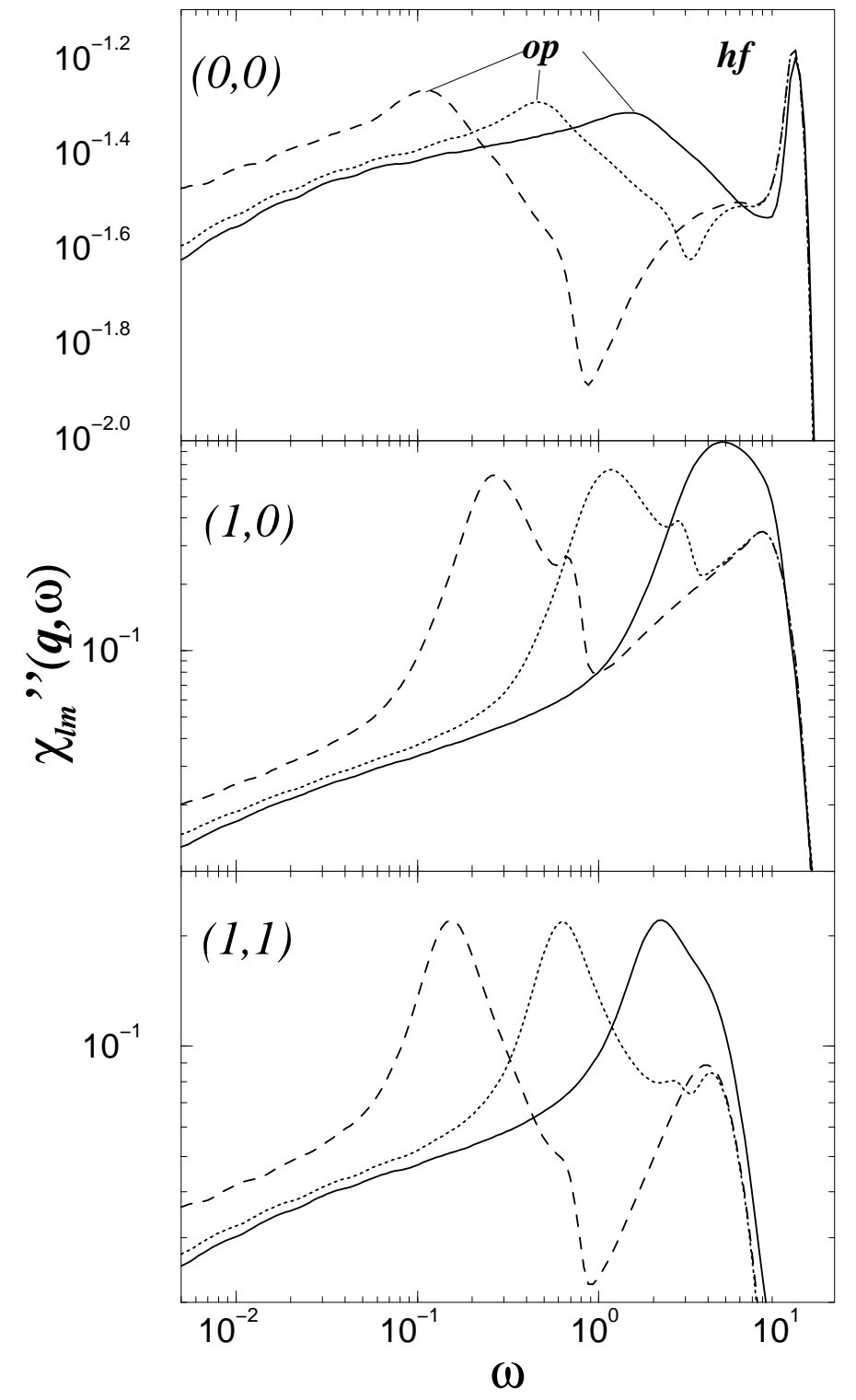

FIG. 12. $\chi_{l m}^{\prime \prime}(q \simeq 4.7, \omega)$ at $\phi=0.381, T^{*}=0.04$ for $I=\frac{1}{10}$ (solid), 1 (dotted) and 10 (dashed) 


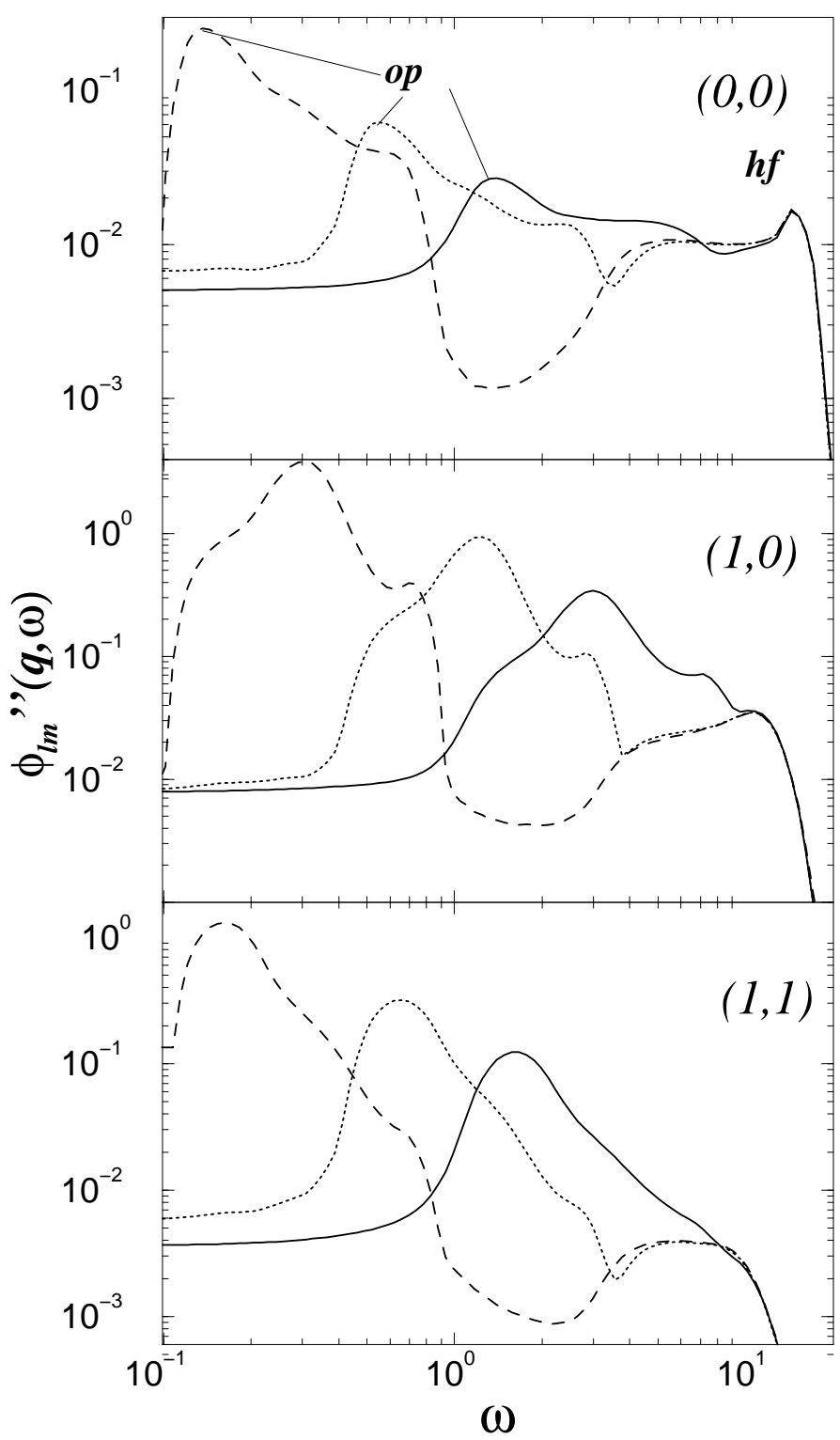

FIG. 13. $\phi_{l m}^{\prime \prime}(q \simeq 4.7, \omega)$ at $\phi=0.53, T^{*}=0.04$ for $I=\frac{1}{10}$ (solid), 1 (dotted) and 10 (dashed)

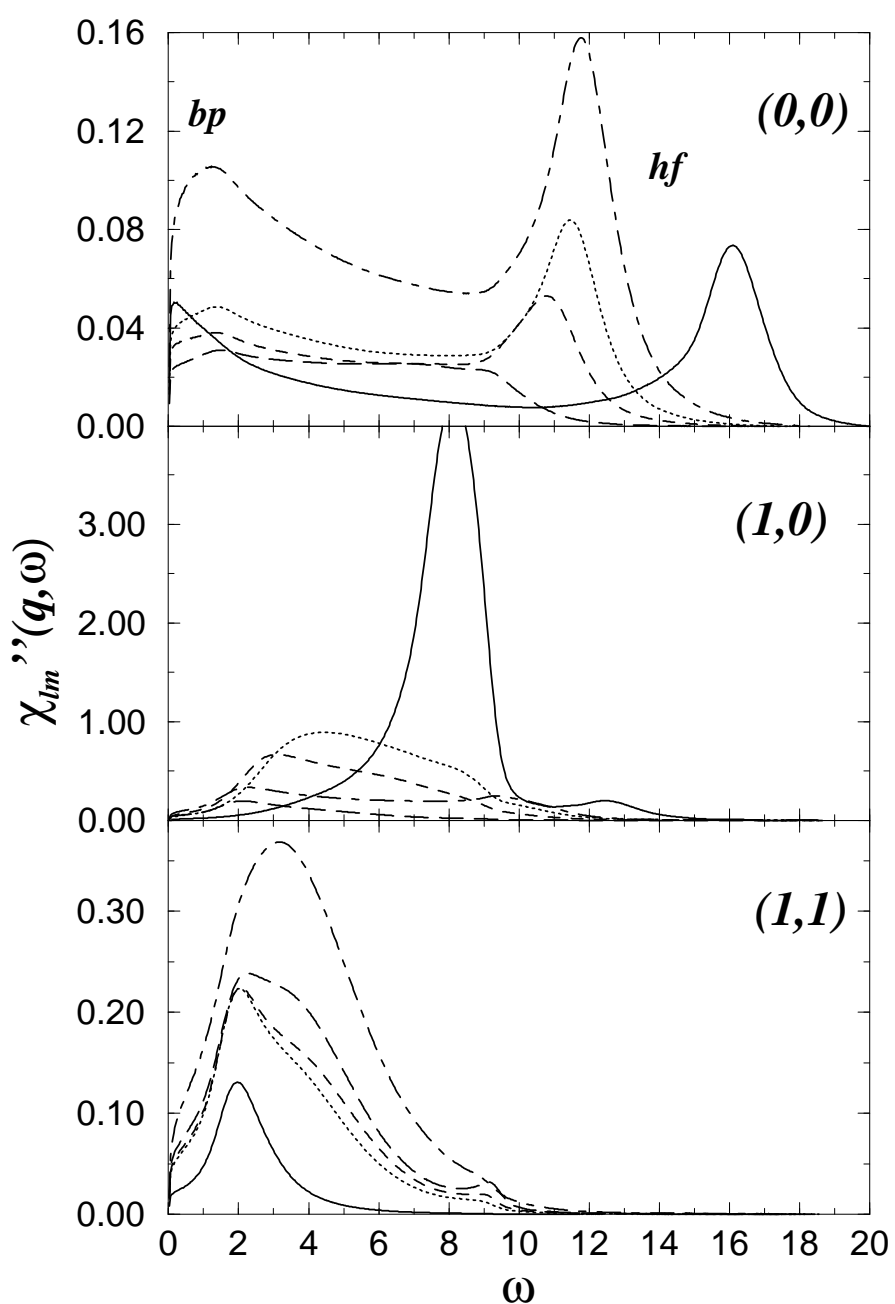

FIG. 14. $q$-dependence of $\chi_{l m}^{\prime \prime}(q, \omega)$ at $\phi=0.381$, $T^{*}=0.04$ for $q \simeq 2.0$ (solid),$q \simeq 4.7$ (dotted),$q \simeq 5.3$ (dashed), $q \simeq 6.5$ (long dashed) and $q \simeq 9.8$ (dot dashed) 


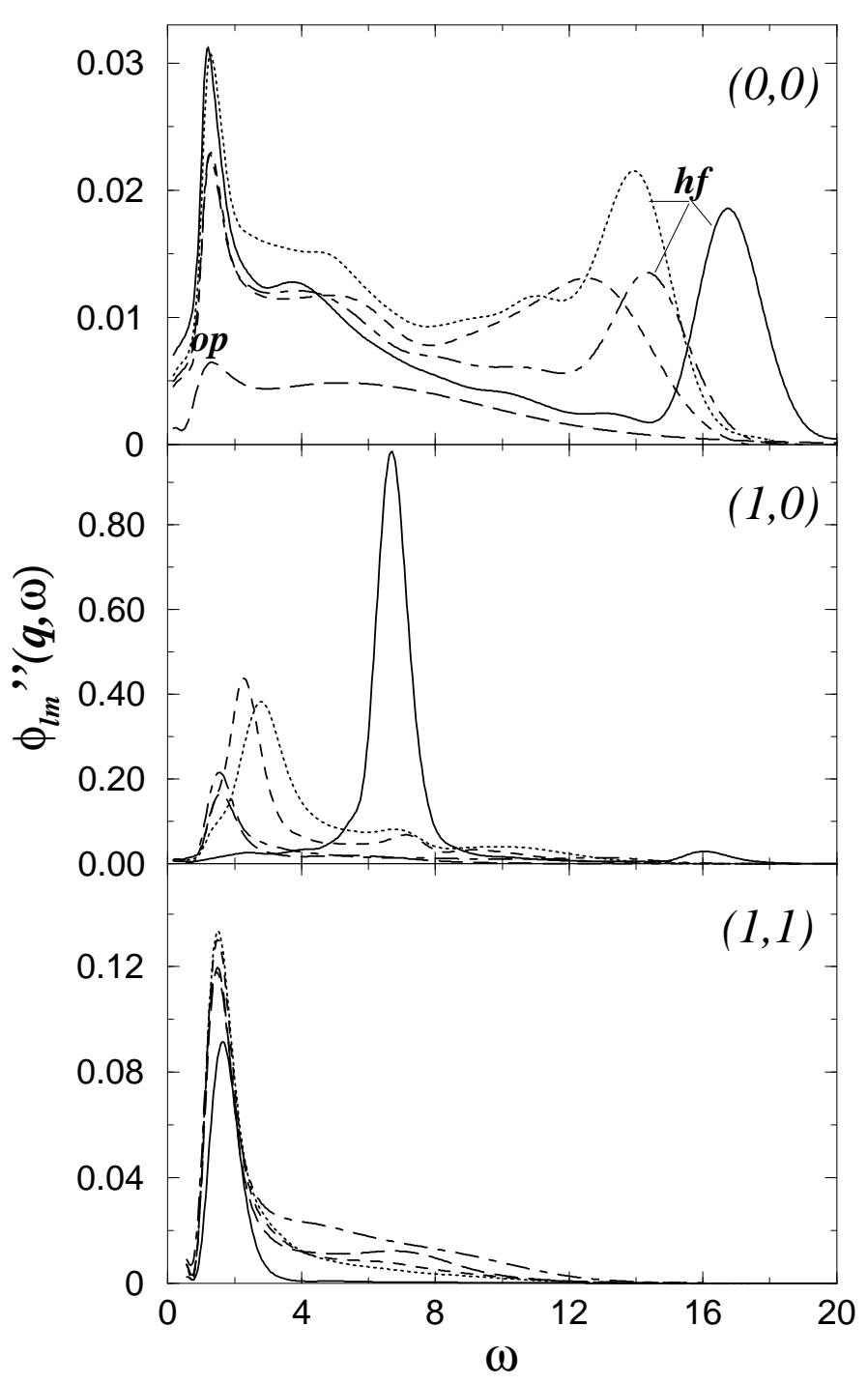

FIG. 15. $q$-dependence of $\phi_{l m}^{\prime \prime}(q, \omega)$ at $\phi=0.53, T^{*}=0.04$ for $q \simeq 2.0$ (solid),$q \simeq 4.7$ (dotted),$q \simeq 5.3$ (dashed),$q \simeq 6.5$ (long dashed) and $q \simeq 9.8$ (dot dashed)

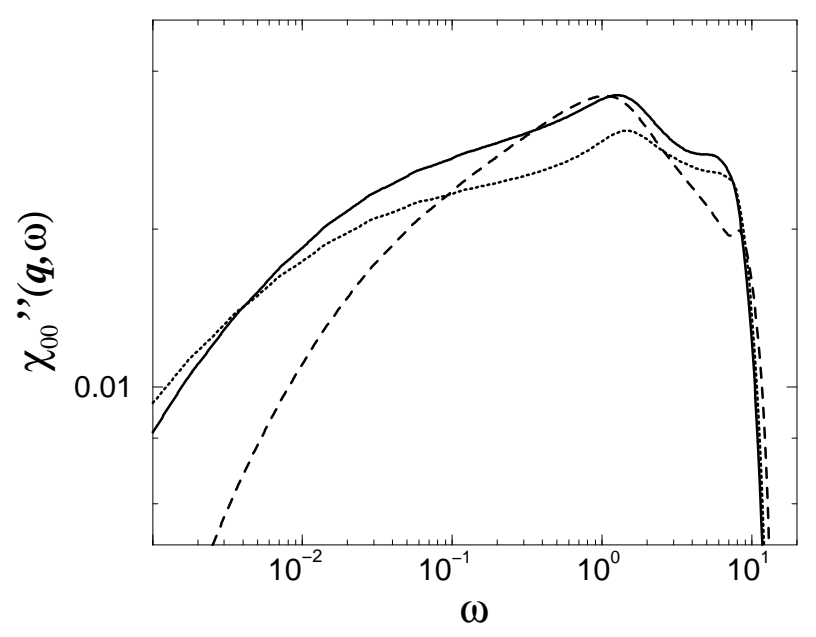

FIG. 16. $\alpha$ - dependence of $\chi_{00}^{\prime \prime}\left(\bar{q}_{\max }, \omega\right)$ at $\phi=0.381$, $T^{*}=0.04$ for $\alpha=0.06$ (dashed), 0.065 (solid) and 0.068 (dotted)

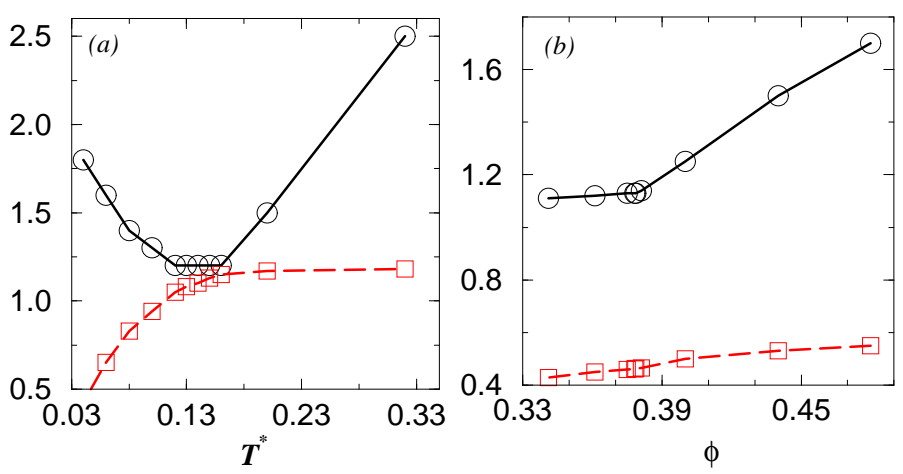

FIG. 17. (a) Temperature dependence of the position $\omega_{o p}$ (o) and height $h_{o p}(\square)$ (multiplied by 10) of the orientational peak for $q \simeq 4.7$ and $\phi_{D}=0.525$ along path D. (b) Dependence on the packing fraction of the position $\omega_{o p}(\circ)$ and height $h_{o p}(\square)$ (multiplied by 10) of the orientational peak for $q \simeq 4.7$ and $T_{A}^{*}=0.04$ along path $\mathrm{A}$

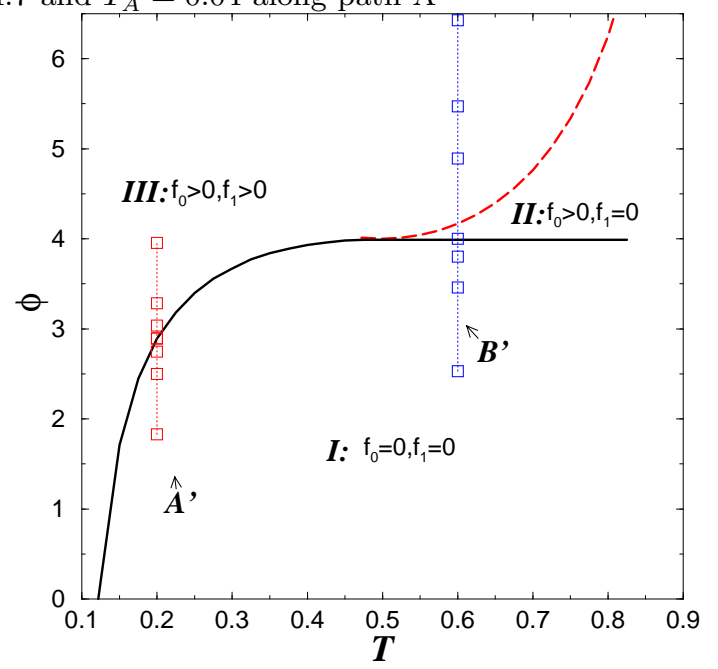

FIG. 18. Phase diagram for the glass transition between the phases I, II and III for the Bosse-Krieger model (59), (60) and (61) with $x_{0}=0.15$. A' and B' denote paths along which we have investigated the dynamics 


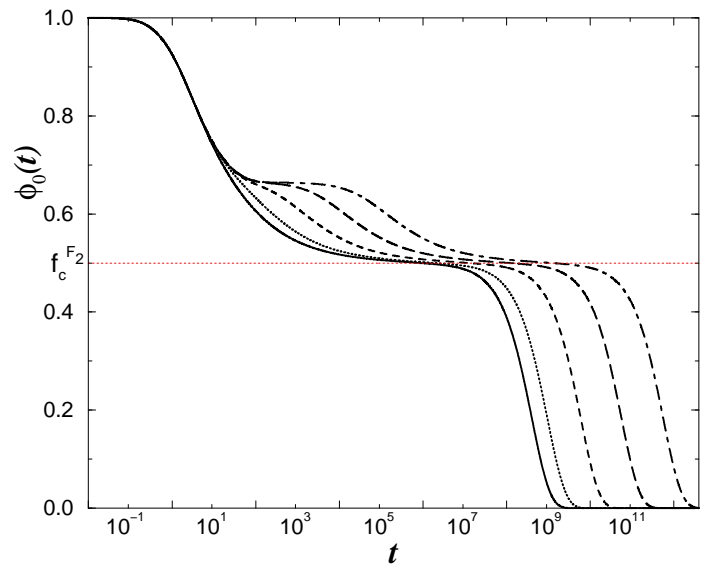

FIG. 19. $\phi_{0}(t)$ for the Bosse-Krieger model for $T=0.7$, $\phi=3.9995, x=0.15, \Omega_{0}=1, \nu_{0}=10, \nu_{1}=1$, and different $\Omega_{1}=10^{-n / 2}, n=0,1,2,3,4$ from left to right. $f_{c}^{F 2}$ denotes the critical non-ergodicity parameter of the $F_{2}$-model

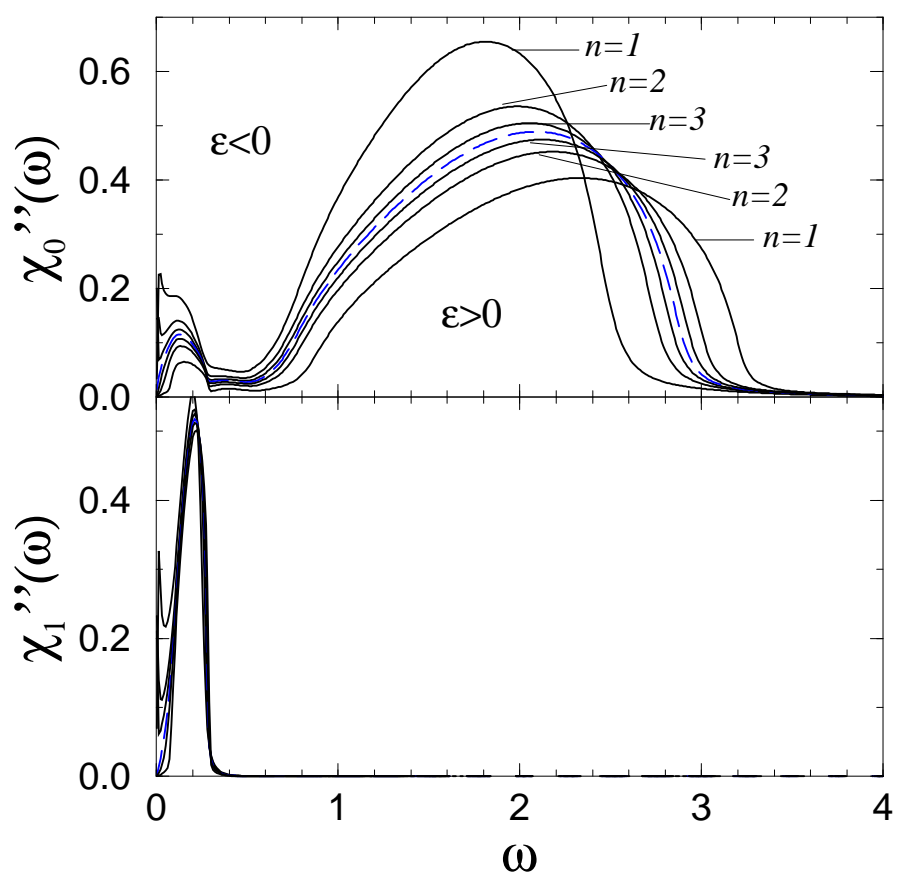

FIG. 20. $\chi_{a}^{\prime \prime}(\omega)$ for $\Omega_{0}=1, \Omega_{1}=1 / 10, \nu_{0}=\nu_{1}=0$, $x=0.15$ and $T=0.2$ along path $\mathrm{A}^{\prime}$ for $\phi=(1+\varepsilon) \phi_{c}$, $\phi_{c} \simeq 2.8931318, \varepsilon= \pm e^{-n}, n=1,2,3$ (solid lines) and $\phi=\phi_{c}$ (dashed line). Because $\chi_{1}^{\prime \prime}(\omega)$ does not vary much with $\phi$ we have not labelled the various curves with $n$

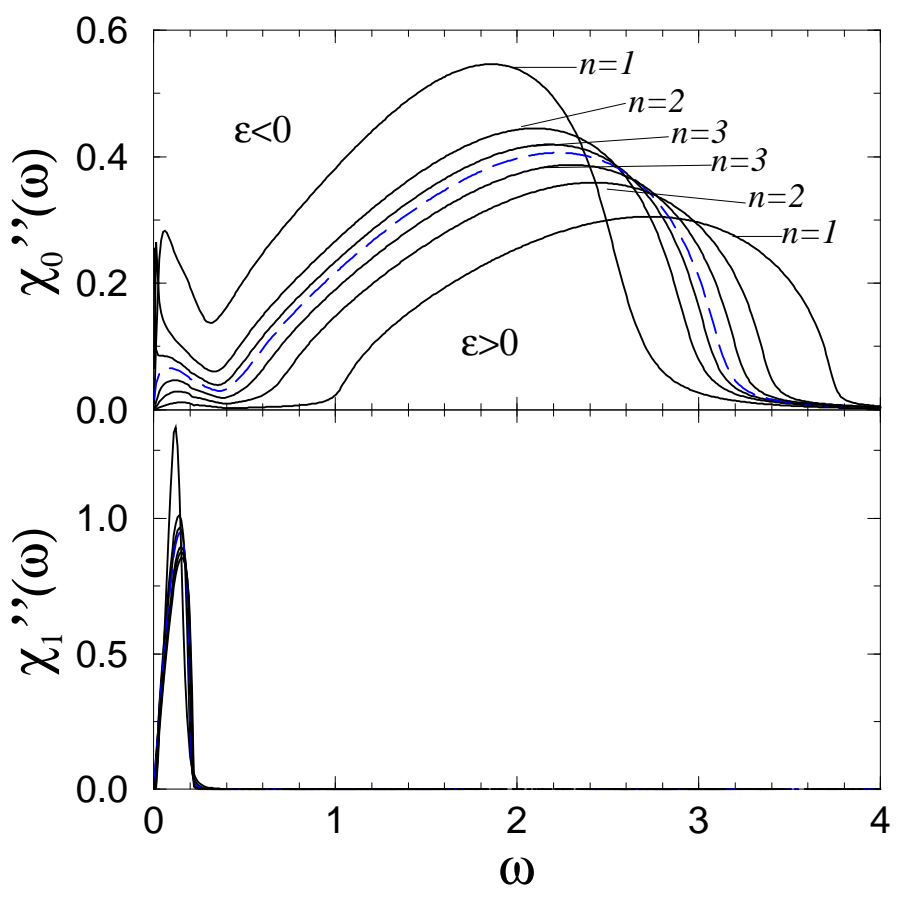

FIG. 21. $\chi_{a}^{\prime \prime}(\omega)$ for $\Omega_{0}=1, \Omega_{1}=1 / 10, \nu_{0}=\nu_{1}=0$, $x=0.15$ and $T=0.6$ along path B' for $\phi=(1+\varepsilon) \phi_{c}, \phi_{c}=4$, $\varepsilon=-e^{-n}$ for $\varepsilon<0$ and $\varepsilon=e^{-n / 2}$ for $\varepsilon>0, n=1,2,3$ (solid lines) and $\phi=\phi_{c}$ (dashed line). Because $\chi_{1}^{\prime \prime}(\omega)$ does not vary much with $\phi$ we have not labelled the various curves with $n$ 


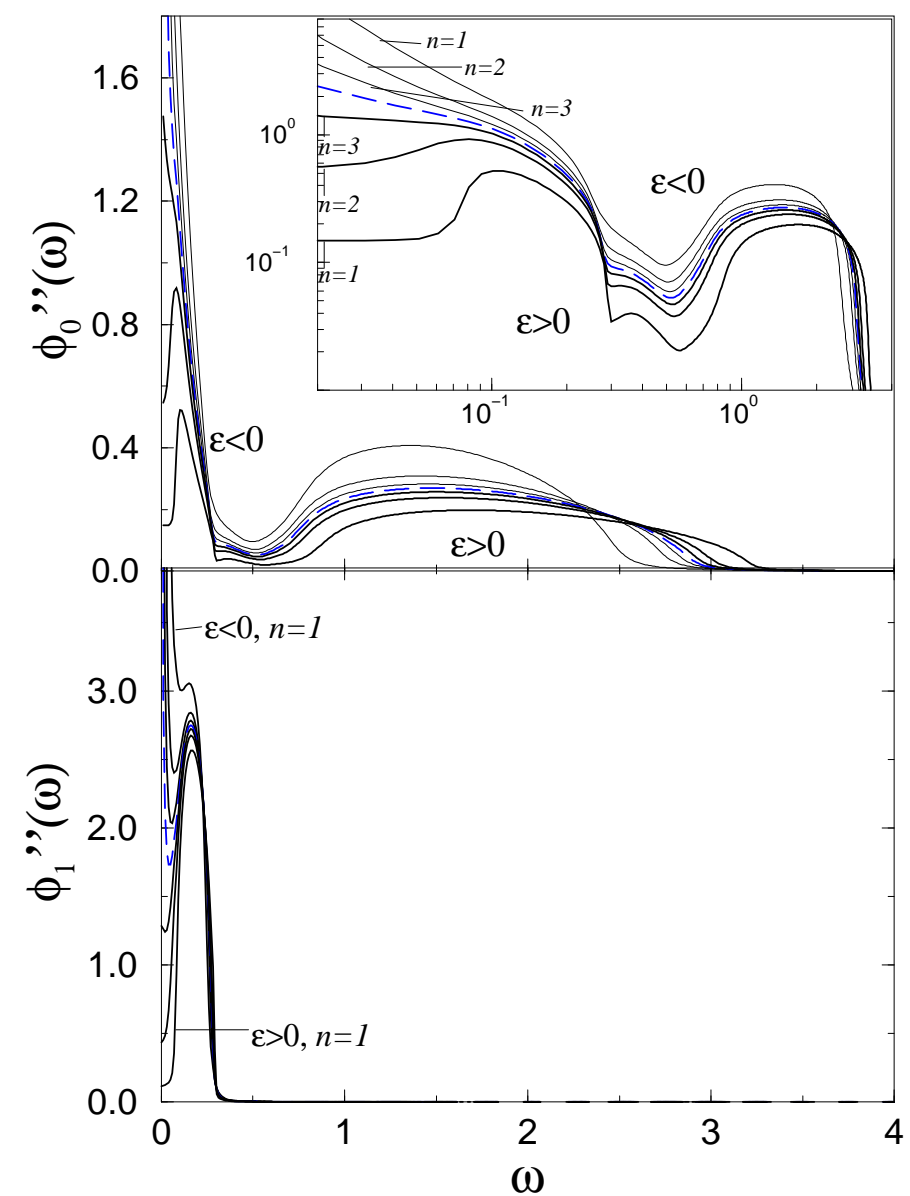

FIG. 22. $\phi_{a}^{\prime \prime}(\omega)$ for $\Omega_{0}=1, \Omega_{1}=1 / 10, \nu_{0}=\nu_{1}=0$, $x=0.15$ and $T=0.2$ along path $\mathrm{A}^{\prime}$ for $\phi=(1+\varepsilon) \phi_{c}$, $\phi_{c} \simeq 2.8931318, \varepsilon= \pm e^{-n}, n=1,2,3$ (solid lines) and $\phi=\phi_{c}$ (dashed line)

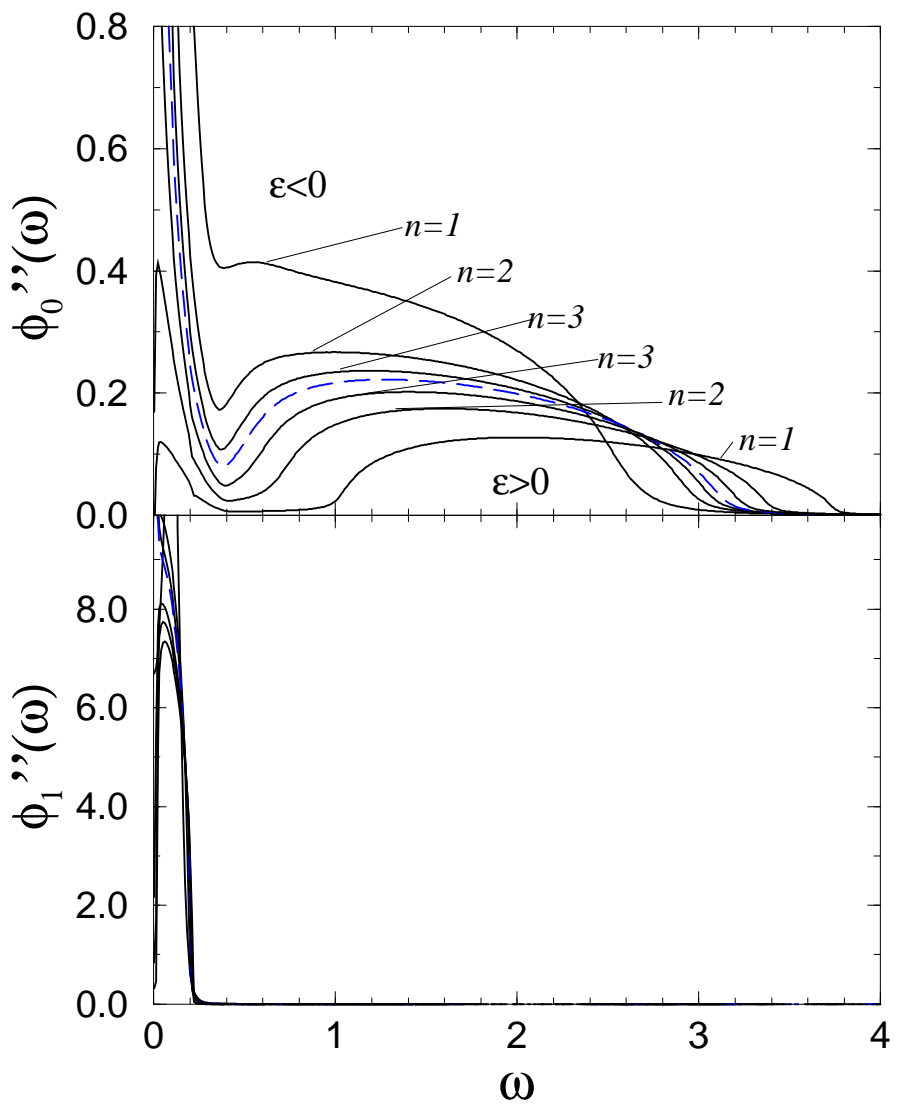

FIG. 23. $\phi_{a}^{\prime \prime}(\omega)$ for $\Omega_{0}=1, \Omega_{1}=1 / 10, \nu_{0}=\nu_{1}=0$, $x=0.15$ and $T=0.6$ along path B' for $\phi=(1+\varepsilon) \phi_{c}, \phi_{c}=4$, $\varepsilon=-e^{-n}$ for $\varepsilon<0$ and $\varepsilon=e^{-n / 2}$ for $\varepsilon>0, n=1,2,3$ (solid lines) and $\phi=\phi_{c}$ (dashed line). Because $\phi_{1}^{\prime \prime}(\omega)$ does not vary much with $\phi$ we have not labelled the various curves with $n$ 


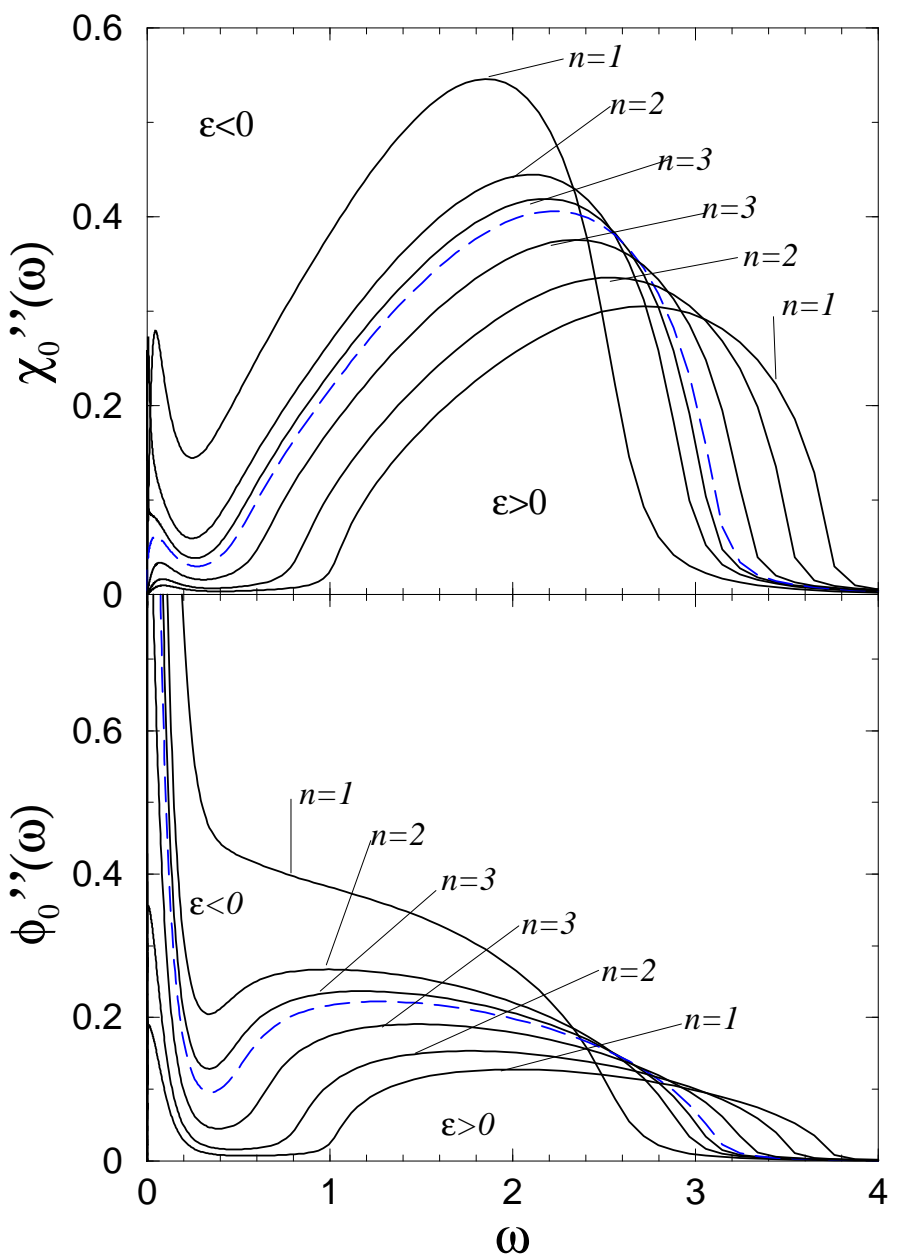

FIG. 24. $\chi_{0}^{\prime \prime}(\omega)$ and $\phi_{0}^{\prime \prime}(\omega)$ for a reduced Bosse-Krieger model $\Omega_{0}=1, \Omega_{1}=1 / 10, \nu_{0}=0, \nu_{1}=0.19, x=0.15$ and $T=0.6 \phi=(1+\varepsilon) \phi_{c}, \phi_{c}=4, \varepsilon=-e^{-n}$, for $\varepsilon<0$ and $\varepsilon=e^{-n / 2}$ for $\varepsilon>0, n=1,2,3$ (solid lines) and $\phi=\phi_{c}$ (dashed line) 